\title{
COMPUTING CODIMENSIONS AND GENERIC CANONICAL FORMS FOR GENERALIZED MATRIX PRODUCTS*
}

\author{
BO KÅGSTRÖM ${ }^{\dagger}$, LARS KARLSSON ${ }^{\dagger}$, AND DANIEL KRESSNER ${ }^{\ddagger}$
}

\begin{abstract}
A generalized matrix product can be formally written as $A_{p}^{s_{p}} A_{p-1}^{s_{p}-1} \cdots A_{2}^{s_{2}} A_{1}^{s_{1}}$, where $s_{i} \in\{-1,+1\}$ and $\left(A_{1}, \ldots, A_{p}\right)$ is a tuple of (possibly rectangular) matrices of suitable dimensions. The periodic eigenvalue problem related to such a product represents a nontrivial extension of generalized eigenvalue and singular value problems. While the classification of generalized matrix products under eigenvalue-preserving similarity transformations and the corresponding canonical forms have been known since the 1970's, finding generic canonical forms has remained an open problem. In this paper, we aim at such generic forms by computing the codimension of the orbit generated by all similarity transformations of a given generalized matrix product. This can be reduced to computing the so called cointeractions between two different blocks in the canonical form. A number of techniques are applied to keep the number of possibilities for different types of cointeractions limited. Nevertheless, the matter remains highly technical; we therefore also provide a computer program for finding the codimension of a canonical form, based on the formulas developed in this paper. A few examples illustrate how our results can be used to determine the generic canonical form of least codimension. Moreover, we describe an algorithm and provide software for extracting the generically regular part of a generalized matrix product.
\end{abstract}

Key words. Matrix product, Periodic eigenvalue problem, Canonical form, Generic Kronecker structure, Cyclic quiver, Orthogonal reduction.

AMS subject classifications. 15A21, 65F 15.

1. Introduction. Consider a matrix pair $\left(A_{1}, A_{2}\right)$, where $A_{1}, A_{2} \in \mathbb{C}^{n_{2} \times n_{1}}$. By the well-known Kronecker canoncial form (KCF) [7], there are square invertible matrices $P_{1}, P_{2}$ such that the transformed pair $\left(P_{2}^{-1} A_{1} P_{1}, P_{2}^{-1} A_{2} P_{1}\right)$ is block diagonal with each diagonal block taking the form

$$
\left(J_{m}(\lambda), I_{m}\right) \text { or }\left(I_{m}, J_{m}(0)\right) \quad \text { or } \quad\left(F_{m}, G_{m}\right) \quad \text { or } \quad\left(F_{m}^{T}, G_{m}^{T}\right)
$$

${ }^{*}$ Received by the editors on July 5, 2010. Accepted for publication on February 27, 2011. Handling Editor: Shmuel Friedland.

${ }^{\dagger}$ Department of Computing Science, Umeå University, Umeå, Sweden (bokg@cs.umu.se, larsk@cs.umu.se). The research was supported by the Swedish Foundation for Strategic Research (grant A3 02:128), and by eSSENCE, a strategic collaborative program in e-Science funded by the Swedish Research Council.

¥Seminar for Applied Mathematics, ETH Zurich, Switzerland (kressner@math.ethz.ch). 
where

$$
J_{m}(\lambda)=\underbrace{\left[\begin{array}{cccc}
\lambda & 1 & & \\
& \lambda & \ddots & \\
& & \ddots & 1 \\
& & & \lambda
\end{array}\right]}_{m \times m}, F_{m}=\underbrace{\left[\begin{array}{cccc}
1 & 0 & & \\
& \ddots & \ddots & \\
& & 1 & 0
\end{array}\right]}_{m \times(m+1)}, G_{m}=\underbrace{\left[\begin{array}{llll}
0 & 1 & & \\
& \ddots & \ddots & \\
& 0 & 1
\end{array}\right]}_{m \times(m+1)}
$$

for some $\lambda \in \mathbb{C}$. Regular blocks take the form $\left(J_{m}(\lambda), I_{m}\right)$ or $\left(I_{m}, J_{m}(0)\right)$, with $m \geq 1$, corresponding to finite or infinite eigenvalues, respectively. Singular blocks take the form $\left(F_{m}, G_{m}\right)$ or $\left(F_{m}^{T}, G_{m}^{T}\right)$, with $m \geq 0$, and correspond to so called Kronecker indices. More specifically, $\left(F_{m}, G_{m}\right)$ corresponds to right indices and $\left(F_{m}^{T}, G_{m}^{T}\right)$ to left indices. The matrix pair $\left(A_{1}, A_{2}\right)$ itself is called singular if its Kronecker form contains at least one singular block.

Identifying the KCF of a singular matrix pair $\left(A_{1}, A_{2}\right)$ is an ill-posed problem. In view of the resulting numerical challenges, it is natural to ask for the most generic forms in the set of all $n_{2} \times n_{1}$ matrix pairs, possibly with additional side constraints on the matrices $A_{1}$ and $A_{2}$. It is well known that the generic form of a square matrix pair, $n=n_{1}=n_{2}$, solely consists of $n$ regular $1 \times 1$ blocks $\left(1, \lambda_{k}\right)$ with $\lambda_{k} \neq \lambda_{j}$ for $k \neq j$. For the subset of square singular matrix pairs, Waterhouse [28] showed that the generic KCFs consist of two singular blocks $\left(F_{j}, G_{j}\right)$ and $\left(F_{n-j+1}^{T}, G_{n-j+1}^{T}\right)$, with $j=1, \ldots, n$. In the rectangular case, $\triangle=n_{1}-n_{2}>0$, there are generically $\left(n_{2}\right.$ $\bmod \triangle)$ blocks $\left(F_{\alpha+1}, G_{\alpha+1}\right)$ with $\alpha=\left\lfloor n_{2} / \triangle\right\rfloor, \triangle-\left(n_{2} \bmod \triangle\right)$ blocks $\left(F_{\alpha}, G_{\alpha}\right)$, and $n o$ regular block $[22,3]$. For the other rectangular case, $\triangle=n_{2}-n_{1}>0$, the result is similar. There are generically $\left(n_{1} \bmod \triangle\right)$ blocks $\left(F_{\alpha+1}^{T}, G_{\alpha+1}^{T}\right)$ with $\alpha=\left\lfloor n_{1} / \triangle\right\rfloor$ and $\triangle-\left(n_{1} \bmod \triangle\right)$ blocks $\left(F_{\alpha}^{T}, G_{\alpha}^{T}\right)$. From these early results on, tremendous progress has been made in understanding and computing less generic KCFs, see [16] for an overview. Recent results include generic KCFs for zero-structured matrix pairs [15] and matrix pairs of fixed normal rank [2].

This paper considers a nontrivial extension of matrix pairs $\left(A_{1}, A_{2}\right)$ (matrix pencils $\left.A_{1}-\lambda A_{2}\right)$. For a fixed sign tuple $s=\left(s_{1}, \ldots, s_{p}\right)$, where $s_{k} \in\{-1,+1\}$, and a fixed dimension tuple $n=\left(n_{1}, n_{2}, \ldots, n_{p}\right)$, let $\mathcal{A}=\left(A_{1}, \ldots, A_{p}\right)$ be a matrix tuple with

$$
A_{k} \in \begin{cases}\mathbb{C}^{n_{k \oplus 1} \times n_{k}} & \text { if } s_{k}=1, \\ \mathbb{C}^{n_{k} \times n_{k \oplus 1}} & \text { if } s_{k}=-1 .\end{cases}
$$

Here and in the following, we let

$$
k \oplus 1:=(k \bmod p)+1
$$

which simply means that $k \oplus 1=k+1$ for $1 \leq k \leq p-1$ and $p \oplus 1=1$. This paper aims at identifying generic canonical forms of such a matrix tuple under the 
equivalence transformation

$$
A_{k} \mapsto \begin{cases}P_{k \oplus 1}^{-1} A_{k} P_{k} & \text { if } s_{k}=1, \\ P_{k}^{-1} A_{k} P_{k \oplus 1} & \text { if } s_{k}=-1,\end{cases}
$$

with invertible matrices $P_{k} \in \mathbb{C}^{n_{k} \times n_{k}}, k=1, \ldots, p$.

A more intuitive understanding of the transformation (1.3) can be gained by regarding the matrix tuple as a generalized matrix product

$$
A_{p}^{s_{p}} A_{p-1}^{s_{p-1}} \cdots A_{1}^{s_{1}}
$$

Note that this matrix product should be understood in a formal sense; we explicitly admit rectangular factors $A_{k}$ for $s_{k}=-1$. If, however, all factors $A_{k}$ with $s_{k}=-1$ are square and invertible, the product (1.4) becomes well-defined in the usual sense and (1.3) corresponds to a similarity transformation of (1.4) and all other products with cyclically permuted factors:

$$
\begin{aligned}
A_{p}^{s_{p}} A_{p-1}^{s_{p-1}} \cdots A_{2}^{s_{2}} A_{1}^{s_{1}} & \mapsto P_{1}^{-1} A_{p}^{s_{p}} A_{p-1}^{s_{p-1}} \cdots A_{2}^{s_{2}} A_{1}^{s_{1}} P_{1}, \\
A_{1}^{s_{1}} A_{p}^{s_{p}} A_{p-1}^{s_{p-1}} \cdots A_{2}^{s_{2}} & \mapsto P_{2}^{-1} A_{1}^{s_{1}} A_{p}^{s_{p}} A_{p-1}^{s_{p-1}} \cdots A_{2}^{s_{2}} P_{2}, \\
\vdots & \\
A_{p-1}^{s_{p-1}} \cdots A_{2}^{s_{2}} A_{1}^{s_{1}} A_{p}^{s_{p}} & \mapsto P_{p}^{-1} A_{p-1}^{s_{p-1}} \cdots A_{2}^{s_{2}} A_{1}^{s_{1}} A_{p}^{s_{p}} P_{p} .
\end{aligned}
$$

A number of applications lead to generalized matrix products of the form (1.4), including periodic control systems [27]. More specifically, applications leading to products with rectangular factors can be found in $[24,26,19]$. The generalized singular value problem for a matrix pair $(A, B)$ can - at least theoretically - be seen as a generalized matrix product eigenvalue problem $A_{4}^{s_{4}} A_{3}^{s_{3}} A_{2}^{s_{2}} A_{1}^{s_{1}}$ with $A_{4}=A^{T}, A_{3}=B^{T}, A_{2}=B$, $A_{1}=A$, and sign tuple $s=(+1,-1,-1,+1)[8,23]$.

As explained by Sergeichuk in [20, 21], canonical forms under the transformation (1.3) were known for a long time in representation theory. In particular, the representations of a quiver associated with (1.4) were classified independently by Nazarova [18] as well as by Donovan and Freislich [6] in the 1970's. Numerical algorithms for extracting the Kronecker-like structure of (1.4) can be found in [21, 25]. They share the difficulties with the GUPTRI algorithm $[4,5]$ in the sense that certain rank decisions have a critical impact on their robustness. These rank decisions can be avoided if we only aim at identifying the generic Kronecker-like structure induced by the dimensions $n_{1}, \ldots, n_{p}$ of the involved factors. For this purpose, we count the codimension of the orbit generated by all similarity transformations of a canonical form, see Section 3. In theory, the most generic canonical form among all possible canonical forms of a given matrix tuple is simply the one of least codimension. In practice, however, the matter is complicated by the sheer number of different canonical tuples 
potentially contributing to the codimension count. In Section 4, we therefore describe generic canonical forms only for the special case $s=(+1, \ldots,+1)$. In Section 5 , we develop an algorithm, based on simple unitary transformations, for extracting the generically regular part of a generalized matrix product. Finally, some conclusions and open questions are summarized in Section 6.

2. Preliminaries. In the following, we consider the sign tuple $\left(s_{1}, \ldots, s_{p}\right)$ to be fixed. We will make use of the following notation. Let $\mathcal{A}=\left(A_{1}, \ldots, A_{p}\right)$ be a matrix tuple with dimensions conforming to (1.2). A tuple $\mathcal{P}=\left(P_{1}, \ldots, P_{p}\right)$ is called a transformation tuple if each $P_{k}$ is $n_{k} \times n_{k}$ and invertible. Then $\mathcal{B}=\left(B_{1}, \ldots, B_{p}\right)=$ $\mathcal{P}\{\mathcal{A}\}$ denotes the equivalence transformation (1.3) with respect to $\mathcal{P}$ :

$$
B_{k}= \begin{cases}P_{k \oplus 1}^{-1} A_{k} P_{k} & \text { if } s_{k}=1 \\ P_{k}^{-1} A_{k} P_{k \oplus 1} & \text { if } s_{k}=-1\end{cases}
$$

The direct sum of two matrix tuples $\mathcal{C}, \mathcal{D}$ is denoted by $\mathcal{C} \oplus \mathcal{D}$ and defined as

$$
\left(\left[\begin{array}{cc}
C_{1} & 0 \\
0 & D_{1}
\end{array}\right], \cdots,\left[\begin{array}{cc}
C_{p} & 0 \\
0 & D_{p}
\end{array}\right]\right) .
$$

As an immediate consequence, the relation

$$
(\mathcal{P} \oplus \mathcal{Q})\{\mathcal{C} \oplus \mathcal{D}\}=\mathcal{P}\{\mathcal{C}\} \oplus \mathcal{Q}\{\mathcal{D}\}
$$

holds provided that the dimensions of $\mathcal{P}$ and $\mathcal{Q}$ conform to the dimensions of $\mathcal{C}$ and $\mathcal{D}$, respectively.

TheOREM 2.1 ([21]). Let $\mathcal{A}=\left(A_{1}, \ldots, A_{p}\right)$ be a matrix tuple of conforming dimensions (1.2). Then there is a transformation tuple $\mathcal{P}$ such that $\mathcal{P}\{\mathcal{A}\}$ can be written as the direct sum of tuples taking one of the following forms.

(i) $\mathcal{J}_{m}(\lambda)=\left(J_{m}(\lambda), \ldots\right)$ with $\lambda \in \mathbb{C} \backslash\{0\}, \quad$ (nonzero, finite Jordan tuple)

(ii) $\mathcal{N}_{m}(k)=\left(\cdots, J_{m}(0), \ldots\right), \quad$ (zero/infinite Jordan tuple with block at pos. $k$ )

(iii) $\mathcal{R}_{m}\left(k_{1}, k_{2}\right)= \begin{cases}\left(\ldots, F_{m}, \ldots, G_{m}, \ldots\right) & \text { if } s_{k_{1}} \neq s_{k_{2}}, \\ \left(\ldots, F_{m}, \ldots, G_{m}^{T}, \ldots\right) & \text { if } s_{k_{1}}=s_{k_{2}},\end{cases}$

(right singular tuple with blocks at pos. $k_{1}$ and $k_{2}$ )

(iv) $\mathcal{L}_{m}\left(k_{1}, k_{2}\right)= \begin{cases}\left(\ldots, F_{m}^{T}, \ldots, G_{m}^{T}, \ldots\right) & \text { if } s_{k_{1}} \neq s_{k_{2}}, \\ \left(\ldots, F_{m}^{T}, \ldots, G_{m}, \ldots\right) & \text { if } s_{k_{1}}=s_{k_{2}},\end{cases}$

(left singular tuple with blocks at pos. $k_{1}$ and $k_{2}$ )

where ... denotes a sequence of identity matrices of appropriate dimension. This decomposition is uniquely determined up to permutation of the summands.

Note that the order of $F_{m}$ and $G_{m}$ in (iii) and (iv) of Theorem 2.1 is not important; the roles of $F_{m}$ and $G_{m}$ can be interchanged by applying a permutation. 
The following linguistic conventions will be useful. The tuples Theorem 2.1.(i) and (ii) are called Jordan tuples and the tuples appearing in Theorem 2.1.(iii) and (iv) are called singular tuples. We say that the Jordan tuple $\mathcal{N}_{m}(k)$ from Theorem 2.1 (ii) belongs to $\lambda=0$ if $s_{k}=1$ and to $\lambda=\infty$ if $s_{k}=-1$. If there are several Jordan tuples belonging to a particular eigenvalue $\lambda$ (may it be nonzero finite, zero, or infinite), we let $q_{j}(\lambda)$ denote the dimension of the $j$ th tuple. That is, the tuple has the form $\mathcal{J}_{q_{j}(\lambda)}(\lambda)$ (if $\lambda$ is nonzero and finite) or $\mathcal{N}_{q_{j}(\lambda)}(k)$ for some $k$ (otherwise). In the following, we will always assume the ordering $q_{1}(\lambda) \geq q_{2}(\lambda) \geq \cdots$. A matrix tuple $\mathcal{A}$ is called regular if its canonical form only contains Jordan tuples, and singular otherwise.

3. Codimension of the tuple orbit. The set of all tuples that can be obtained from $\mathcal{A}$ by an equivalence transformation (1.3) forms a manifold in the space $\mathbb{C}^{N}$, where $N=\sum_{k=1}^{p} n_{k} n_{k \oplus 1}$. In the definition of $N$, each $A_{k}$ contributes $n_{k} n_{k \oplus 1}$ to the dimension of the manifold. This manifold will be denoted by

$$
\operatorname{orbit}(\mathcal{A})=\{\mathcal{P}\{\mathcal{A}\}: \mathcal{P} \text { is a transformation tuple }\}
$$

The dimension of an orbit is the dimension of its tangent space at a specified tuple in the manifold, and the codimension is the dimension of the normal space of the orbit at the same specified tuple. Trivially, the dimension and the codimension of the orbit add up to $N$. The aim of this section is to count the codimension of this manifold in terms of the generalized Kronecker structure of $\mathcal{A}$. More specifically, Table 3.1 provides a summary of the main result we are aiming at and the rest of this section is devoted to proving and filling in the details of Table 3.1.

3.1. Breakdown into cointeractions. We will follow the strategy of Demmel and Edelman [3] to compute the codimension as the sum of cointeractions between simple building blocks of the tangent space of $\operatorname{orbit}(\mathcal{A})$ at $\mathcal{A}$. To compute this tangent space, we choose the transformation matrices $P_{k}=I+\delta X_{k}$ for sufficiently small $\delta$. Note that $P_{k}^{-1}=\left(I+\delta X_{k}\right)^{-1}=I-\delta X_{k}+O\left(\delta^{2}\right)$. This shows that - to first order - the elements of the correspondingly transformed tuple $\mathcal{P}\{\mathcal{A}\}$ take the form $A_{k}+\delta\left(A_{k} X_{k}-X_{k \oplus 1} A_{k}\right)$ if $s_{k}=1$ and $A_{k}+\delta\left(A_{k} X_{k \oplus 1}-X_{k} A_{k}\right)$ if $s_{k}=-1$. Therefore, the tangent space of $\operatorname{orbit}(\mathcal{A})$ at $\mathcal{A}$ consists of matrix tuples in the image of the linear operator

$$
\text { Sylv }: \mathcal{X} \mapsto \mathcal{Y} \quad \text { with } \quad Y_{k}= \begin{cases}A_{k} X_{k}-X_{k \oplus 1} A_{k} & \text { if } s_{k}=1 \\ A_{k} X_{k \oplus 1}-X_{k} A_{k} & \text { if } s_{k}=-1\end{cases}
$$

Note that the matrices $X_{k}$ are $n_{k} \times n_{k}$ but the matrices $Y_{k}$ are $n_{k \oplus 1} \times n_{k}$ for $s_{k}=1$, or $n_{k} \times n_{k \oplus 1}$ for $s_{k}=-1$. If $d$ denotes the dimension of the kernel of the linear 
The codimension of the orbit of the tuple $\mathcal{A}$ only depends on its canonical form described by Theorem 2.1. It can be computed as the sum

$$
c_{\text {total }}=c_{\text {Jordan }}+c_{\text {singular }}+c_{\text {Jordan, singular }}
$$

with the summands defined as follows.

1. $c_{\text {Jordan }}$ is the sum of cointeractions between Jordan tuples:

$$
c_{\text {Jordan }}=\sum_{\lambda} q_{1}(\lambda)+3 q_{2}(\lambda)+5 q_{3}(\lambda)+\cdots,
$$

where the sum is taken over all eigenvalues $\lambda$ of $\mathcal{A}$, including zero and infinite eigenvalues, and $q_{j}(\lambda)$ denotes the decreasingly ordered dimensions of each Jordan tuple belonging to $\lambda$;

2. $c_{\text {singular }}$ is the sum of cointeractions between singular tuples described in Section 3.3;

3. $c_{\text {Jordan, singular }}$ is the sum of cointeractions between Jordan tuples and singular tuples described in Section 3.4.

Summary of the main result on counting codimensions.

operator Sylv, then the codimension of $\operatorname{orbit}(\mathcal{A})$ is given by

$$
\sum_{k=1}^{p} n_{k} n_{k \oplus 1}-\left(\sum_{k=1}^{p} n_{k}^{2}-d\right)=d-\frac{1}{2} \sum_{k=1}^{p}\left(n_{k \oplus 1}-n_{k}\right)^{2}
$$

The term $\frac{1}{2} \sum_{k=1}^{p}\left(n_{k \oplus 1}-n_{k}\right)^{2}$ counts the differences in the dimensions of $A_{k}$ and disappears if all factors are square. It remains to determine $d$.

It is not hard to see that the dimension of the kernel of Sylv does not change when $\mathcal{A}$ undergoes an equivalence transformation by a transformation tuple $\mathcal{P}$, see (1.3). We can therefore assume without loss of generality that $\mathcal{A}$ is in the canonical form described by Theorem 2.1. To break down the dimension count into the individual tuples in this canonical form, we first investigate the direct sum of two tuples: $\mathcal{A}=$ $\mathcal{B} \oplus \mathcal{C}$. Let us partition $X_{k}=\left[\begin{array}{cc}X_{11}^{(k)} & X_{12}^{(k)} \\ X_{21}^{(k)} & X_{22}^{(k)}\end{array}\right]$ conformally such that (3.1) can be written as

$$
A_{k} X_{k}-X_{k \oplus 1} A_{k}=\left[\begin{array}{cc}
B_{k} X_{11}^{(k)}-X_{11}^{(k \oplus 1)} B_{k} & B_{k} X_{12}^{(k)}-X_{12}^{(k \oplus 1)} C_{k} \\
C_{k} X_{21}^{(k)}-X_{21}^{(k \oplus 1)} B_{k} & C_{k} X_{22}^{(k)}-X_{22}^{(k \oplus 1)} C_{k}
\end{array}\right]
$$

for $s_{k}=1$, and analogously for $s_{k}=-1$. The number $d$ of linearly independent solutions to $A_{k} X_{k}-X_{k \oplus 1} A_{k}=0$ is the sum of the number of linearly independent 
solutions to each of the four (decoupled) subblocks. We therefore obtain

$$
d=d(\mathcal{B}, \mathcal{B})+d(\mathcal{B}, \mathcal{C})+d(\mathcal{C}, \mathcal{B})+d(\mathcal{C}, \mathcal{C})
$$

where $d(\mathcal{B}, \mathcal{C})$ is the dimension of the kernel of the linear operator

$$
\operatorname{Sylv}(\mathcal{B}, \mathcal{C}): \mathcal{X} \mapsto \mathcal{Y} \quad \text { with } \quad Y_{k}= \begin{cases}B_{k} X_{k}-X_{k \oplus 1} C_{k} & \text { if } s_{k}=1 \\ B_{k} X_{k \oplus 1}-X_{k} C_{k} & \text { if } s_{k}=-1\end{cases}
$$

and the other quantities in (3.2) are analogously defined. This discussion extends in a straightforward way to direct sums of more than two tuples. Following [3], we introduce the concepts of interactions and cointeractions between the individual components of a direct sum.

Definition 3.1. Let $\mathcal{A}=\mathcal{B}_{1} \oplus \cdots \oplus \mathcal{B}_{t}$ with each tuple $\mathcal{B}_{j}$ having dimensions $\left(n_{1}^{(j)}, n_{2}^{(j)}, \ldots, n_{p}^{(j)}\right)$. Then the interaction $d\left(\mathcal{B}_{i}, \mathcal{B}_{j}\right)$ between $\mathcal{B}_{i}$ and $\mathcal{B}_{j}$ is the dimension of the kernel of $\operatorname{Sylv}\left(\mathcal{B}_{i}, \mathcal{B}_{j}\right)$ defined in (3.3). The cointeraction between $\mathcal{B}_{i}$ and $\mathcal{B}_{j}$ is defined as

$$
c\left(\mathcal{B}_{i}, \mathcal{B}_{j}\right):=d\left(\mathcal{B}_{i}, \mathcal{B}_{j}\right)-\frac{1}{2} \sum_{k=1}^{p}\left(n_{k \oplus 1}^{(i)}-n_{k}^{(i)}\right)\left(n_{k \oplus 1}^{(j)}-n_{k}^{(j)}\right)
$$

A direct extension of (3.2) shows that $d$ is the sum of all possible interactions, yielding the following result.

LEMma 3.2. The codimension of the orbit of $\mathcal{A}=\mathcal{B}_{1} \oplus \cdots \oplus \mathcal{B}_{t}$ is the sum of cointeractions $c\left(\mathcal{B}_{i}, \mathcal{B}_{j}\right)$ for all combinations of $i$ and $j$ with $i, j \in[1, t]$.

Proof. The result follows from

$$
\begin{aligned}
\sum_{i, j=1}^{t} c\left(\mathcal{B}_{i}, \mathcal{B}_{j}\right) & =\sum_{i, j=1}^{t} d\left(\mathcal{B}_{i}, \mathcal{B}_{j}\right)-\frac{1}{2} \sum_{i, j=1}^{t} \sum_{k=1}^{p}\left(n_{k \oplus 1}^{(i)}-n_{k}^{(i)}\right)\left(n_{k \oplus 1}^{(j)}-n_{k}^{(j)}\right) \\
& =d-\frac{1}{2} \sum_{k=1}^{p} \sum_{i, j=1}^{t}\left(n_{k \oplus 1}^{(i)}-n_{k}^{(i)}\right)\left(n_{k \oplus 1}^{(j)}-n_{k}^{(j)}\right) \\
& =d-\frac{1}{2} \sum_{k=1}^{p}\left[\sum_{i=1}^{t}\left(n_{k \oplus 1}^{(i)}-n_{k}^{(i)}\right)\right]^{2}=d-\frac{1}{2} \sum_{k=1}^{p}\left(n_{k \oplus 1}-n_{k}\right)^{2}
\end{aligned}
$$

In particular, when $\mathcal{A}$ is in canonical form, Lemma 3.2 reveals that counting all cointeractions between canonical tuples yields the codimension of its orbit. In the following, it will be more convenient to count the interactions and obtain the cointeractions according to Definition 3.1. 
3.2. Interactions between Jordan tuples. First, we count interactions between Jordan tuples. For this purpose, the following proposition turns out to be helpful.

Proposition 3.3. Consider the tuples

$$
\mathcal{B}=(\ldots, \underset{\substack{\uparrow \\ k}}{B,}, \ldots), \quad \mathcal{C}=(\ldots, \underset{\uparrow}{C,}, \ldots),
$$

with matrices $B$ and $C$ at positions $k$ and $l$, respectively, and otherwise containing identities. Then

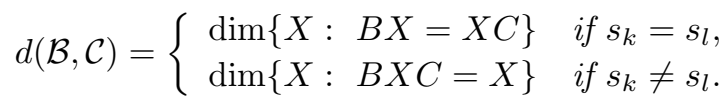

Proof. By shifting the indices, we may assume without loss of generality that $k=1$. Assume $s_{k}=s_{l}=1$. Then $d(\mathcal{B}, \mathcal{C})$ is the kernel dimension of the operator $(3.3)$. Any element $\mathcal{X}=\left(X_{1}, \ldots, X_{p}\right)$ in this kernel satisfies

$$
B X_{1}=X_{2}, X_{2}=X_{3}, \ldots, X_{l-1}=X_{l}, X_{l}=X_{l+1} C, X_{l+1}=X_{l+2}, \ldots, X_{p}=X_{1} \text {. }
$$

Removing all trivial dependencies yields the equation $B X_{1}=X_{1} C$ and hence $d(\mathcal{B}, \mathcal{C})$ is solely determined by the kernel dimension of this equation. The proof for $s_{k}=s_{l}=$ -1 is entirely analogous.

Now assume $s_{k}=1$ but $s_{l}=-1$. Then (3.5) needs to be replaced by

$$
B X_{1}=X_{2}, X_{2}=X_{3}, \ldots, X_{l-1}=X_{l}, X_{l} C=X_{l+1}, X_{l+1}=X_{l+2}, \ldots, X_{p}=X_{1} \text {. }
$$

Removing all trivial dependencies yields the equation $B X_{1} C=X_{1}$. Again, the proof for $s_{k_{1}}=-1, s_{k_{2}}=1$ is analogous.

Lemma 3.1 in [9] on the unique solvability of periodic Sylvester equations implies that the kernels of $\operatorname{Sylv}\left(\mathcal{J}_{\ell}(\lambda), \mathcal{J}_{m}(\mu)\right)$ for $\lambda \neq \mu, \operatorname{Sylv}\left(\mathcal{J}_{\ell}(\lambda), \mathcal{N}_{m}(k)\right)$ and $\operatorname{Sylv}\left(\mathcal{N}_{\ell}(k)\right.$, $\left.\mathcal{J}_{m}(\lambda)\right)$ are all trivial. In the consideration of interactions between Jordan tuples, we can therefore restrict the cointeraction counts to the cases $\left(\mathcal{J}_{\ell}(\lambda), \mathcal{J}_{m}(\lambda)\right)$ and $\left(\mathcal{N}_{\ell}\left(k_{1}\right), \mathcal{N}_{m}\left(k_{2}\right)\right)$.

LEMMA 3.4. $c\left(\mathcal{J}_{\ell}(\lambda), \mathcal{J}_{m}(\lambda)\right)=\min \{\ell, m\}$.

Proof. By Proposition 3.3, $d\left(\mathcal{J}_{\ell}(\lambda), \mathcal{J}_{m}(\lambda)\right)$ is the dimension of the linear space of all matrices satisfying the Sylvester equation $J_{\ell}(\lambda) X-X J_{m}(\lambda)=0$. The statement of the lemma now follows from standard results, see also Proposition A.1.1.

The following result shows not only that the statement of Lemma 3.4 extends to interactions between Jordan tuples belonging to $\lambda \in\{0, \infty\}$, but also that there 
are no interactions between a Jordan tuple belonging to $\lambda=0$ and a Jordan tuple belonging to $\lambda=\infty$.

LEMMA 3.5. $c\left(\mathcal{N}_{\ell}\left(k_{1}\right), \mathcal{N}_{m}\left(k_{2}\right)\right)=\min \{\ell, m\}$ if $s_{k_{1}}=s_{k_{2}}$ and $c\left(\mathcal{N}_{\ell}\left(k_{1}\right), \mathcal{N}_{m}\left(k_{2}\right)\right)$ $=0$ if $s_{k_{1}} \neq s_{k_{2}}$.

Proof. If $s_{k_{1}}=s_{k_{2}}$, then the result follows as in the proof of Lemma 3.4. If $s_{k_{1}} \neq s_{k_{2}}$, then Proposition 3.3 implies that $c\left(\mathcal{N}_{\ell}\left(k_{1}\right), \mathcal{N}_{m}\left(k_{2}\right)\right)$ is the dimension of the linear space of all matrices satisfying the Stein equation $N_{\ell} X_{1} N_{m}-X_{1}=0$. The statement of the lemma now follows from Proposition A.1.3.

Let us summarize the interactions between Jordan tuples. If $\lambda$ is an eigenvalue (may it be finite, zero, or infinite) with Jordan blocks of sizes $q_{1}(\lambda) \geq q_{2}(\lambda) \geq q_{3}(\lambda) \geq$ $\cdots$, then the sum of all cointeractions caused by $\lambda$ is given by

$$
c_{\text {Jordan }}(\lambda)=q_{1}(\lambda)+3 q_{2}(\lambda)+5 q_{3}(\lambda)+\cdots,
$$

just as in the case of standard Jordan canonical and Kronecker canonical forms [3]. The sum of all cointeractions between Jordan tuples is therefore

$$
c_{\text {Jordan }}=\sum_{\lambda} q_{1}(\lambda)+3 q_{2}(\lambda)+5 q_{3}(\lambda)+\cdots
$$

which proves the first item in Table 3.1.

3.3. Interactions between singular tuples. For tuples of the form

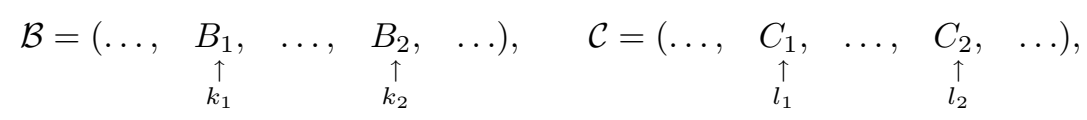

we will see that the periodic Sylvester equation (3.3) reduces to different types of equations, depending on the relative placement of the nontrivial blocks and the sign tuple. There is an overwhelming number of possibilities for the placement and signs. In the following, we discuss several techniques to reduce this number and tame the classification to a certain extent.

Excluding shared positions and assuming $p=4$ w.l.o.g. First, we show how to exclude the special case when the nontrivial coefficients in $\mathcal{B}$ and $\mathcal{C}$ share a position. This is achieved by constructing tuples having the same interaction but no shared position.

Consider first the case $k_{1}=l_{1}$ and $s_{k_{1}}=1$. Then the kernel of the Sylvester operator (3.3) satisfies the equation

$$
B_{1} X_{k_{1}}=X_{k_{1} \oplus 1} C_{1}
$$


By introducing a slack matrix $\tilde{X}$, we obtain the two equations

$$
B_{1} X_{k_{1}}=\tilde{X}, \quad \tilde{X}=X_{k_{1} \oplus 1} C_{1},
$$

corresponding to the extended $(p+1)$-tuples

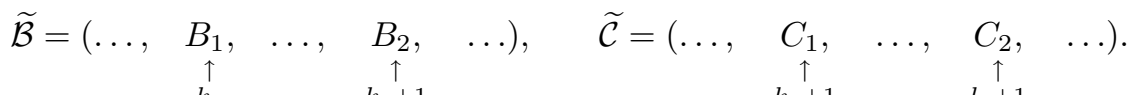

$$
\begin{aligned}
& \begin{array}{llll}
k_{1} & k_{2}+1 & k_{1}+1 & l_{2}+1
\end{array}
\end{aligned}
$$

As the slack matrix does not change the dimension of the kernel of the periodic Sylvester operator, the interactions of the original and the extended tuples are the same. However, the terms of the subtracted sum in (3.4) change, meaning that the cointeractions may change after the slack matrix has been introduced.

Similarly, for $k_{1}=l_{1}$ and $s_{k_{1}}=-1$ the corresponding matrix equation

$$
B_{1} X_{k_{1} \oplus 1}=X_{k_{1}} C_{1}
$$

can be extended such that $B_{1}$ is moved one position to the right, without changing their interaction but possibly changing their cointeraction.

We may therefore assume without loss of generality that $k_{i} \neq l_{j}$ for $i, j=1,2$. Furthermore, by an argument used in the proof of Proposition 3.3, identities at the same position in both tuples correspond to trivial matrix equations that can be removed. We can therefore assume without loss of generality that $\mathcal{B}$ and $\mathcal{C}$ are both tuples of $p=4$ matrices.

Taming 384 cases. Now assuming $p=4$ and no shared position there are precisely six different possibilities for the placement of the blocks in $\mathcal{B}$ and $\mathcal{C}$, as illustrated in the first row of Table 3.2. When taking into account the 16 possible sign combinations, see the first column of Table 3.2, we get 96 different equations in total. Even worse, since each tuple can correspond either to a left or a right singular tuple, there are four different combinations of coefficients in these equations. Hence, in total there would be 384 different cases for which we have to compute the corresponding interaction. Fortunately, many of these cases are equivalent, which helps reduce the computation significantly.

Omitting trivially satisfied parts of the matrix equations, it turns out that there are only eight genuinely different types of matrix equations associated with the 384 different cases, see Table 3.3. It is important to note that the coefficients $\tilde{B}_{1}, \tilde{B}_{2}, \tilde{C}_{1}, \tilde{C}_{2}$ of the matrix equations do not necessarily have the same order as the coefficients $B_{1}, B_{2}, C_{1}, C_{2}$ in $\mathcal{B}, \mathcal{C}$ and need to be adjusted according to the matrix equation variant, see Table 3.4. Note that the symbols $\mathcal{R}_{\ell}$ and $\mathcal{L}_{m}$ are used to denote right and left singular tuples, see Theorem 2.1 . 


\begin{tabular}{|c|c|c|c|c|c|c|}
\hline $\begin{array}{l}\text { sign } \\
\text { tuple }\end{array}$ & \multicolumn{2}{|c|}{$\begin{array}{ll}\left(B_{1}, I, B_{2}, I\right) & \left(I, B_{1}, I, B_{2}\right) \\
\left(I, C_{1}, I, C_{2}\right) & \left(C_{1}, I, C_{2}, I\right)\end{array}$} & \multicolumn{2}{|c|}{$\begin{array}{l}\left(B_{1}, B_{2}, I, I\right)\left(I, I, B_{1}, B_{2}\right) \\
\left(I, \quad I, C_{1}, C_{2}\right)\left(C_{1}, C_{2}, I, I\right)\end{array}$} & \multicolumn{2}{|c|}{$\begin{array}{ll}\left(B_{1}, I, I, B_{2}\right) & \left(I, B_{1}, B_{2}, I\right) \\
\left(I, C_{1}, C_{2}, I\right) & \left(C_{1}, I, I, C_{2}\right)\end{array}$} \\
\hline++++ & VIII: 1 & VIII:3 & $I: 4$ & $I: 4$ & $I: 3$ & $I: 2$ \\
\hline+++- & II : 1 & IV : 1 & II $: 2$ & IV $: 3$ & IV $: 3$ & II $: 2$ \\
\hline++-+ & IV $: 3$ & II $: 2$ & II $: 2$ & IV $: 3$ & II $: 1$ & IV $: 1$ \\
\hline++-- & $\mathrm{V}: 1$ & $\mathrm{~V}: 1$ & III:2 & III : 4 & VII : 1 & $\mathrm{VI}: 1$ \\
\hline+-++ & II $: 2$ & IV $: 3$ & IV $: 3$ & II $: 2$ & II $: 1$ & IV $: 1$ \\
\hline+-+- & III: 2 & III : 4 & $\mathrm{~V}: 1$ & $\mathrm{~V}: 1$ & $\mathrm{~V}: 1$ & $\mathrm{~V}: 1$ \\
\hline+--+ & $\mathrm{V}: 1$ & $\mathrm{~V}: 1$ & $\mathrm{VI}: 1$ & VII : 1 & III : 2 & III $: 1$ \\
\hline+--- & IV $: 3$ & II $: 2$ & IV $: 1$ & II : 1 & IV : 1 & II : 1 \\
\hline-+++ & IV $: 1$ & II $: 1$ & IV $: 3$ & II $: 2$ & IV $: 3$ & II : 2 \\
\hline-++- & $\mathrm{V}: 1$ & $\mathrm{~V}: 1$ & VII : 1 & $\mathrm{VI}: 1$ & III $: 4$ & III: 2 \\
\hline-+-+ & III: 1 & III: 2 & $\mathrm{~V}: 1$ & $\mathrm{~V}: 1$ & $\mathrm{~V}: 1$ & $\mathrm{~V}: 1$ \\
\hline-+-- & II $: 1$ & IV : 1 & IV $: 1$ & II : 1 & II $: 2$ & IV $: 3$ \\
\hline--++ & $\mathrm{V}: 1$ & $\mathrm{~V}: 1$ & III: 1 & III: 2 & $\mathrm{VI}: 1$ & VII: 1 \\
\hline--+- & IV $: 1$ & II $: 1$ & II : 1 & IV $: 1$ & II $: 2$ & IV $: 3$ \\
\hline---+ & II $: 2$ & IV $: 3$ & II $: 1$ & IV $: 1$ & IV $: 1$ & II $: 1$ \\
\hline---- & VIII:3 & VIII: 1 & $\mathrm{I}: 1$ & $I: 1$ & $I: 2$ & $I: 3$ \\
\hline
\end{tabular}

Given singular tuples $\mathcal{B}, \mathcal{C}$ and a sign tuple $s=( \pm 1, \pm 1, \pm 1, \pm 1)$ this table gives the corresponding matrix equation type and variant. For example, IV:3 refers to matrix equation type IV and variant 3.

It is best to illustrate the procedure to obtain the matrix equation and the interaction by a concrete example.

EXAmple 3.6. Consider $s=(+1,+1,-1,+1)$ and

$$
\mathcal{B}=\mathcal{R}_{\ell}(2,4)=\left(I, F_{\ell}, I, G_{\ell}^{T}\right), \quad \mathcal{C}=\mathcal{R}_{m}(1,3)=\left(F_{m}, I, G_{m}, I\right) .
$$

The corresponding matrix equations satisfied by the kernel of the operator (3.3) are $X_{1}=X_{2} F_{m}, F_{\ell} X_{2}=X_{3}, X_{4}=X_{3} G_{m}, G_{\ell}^{T} X_{4}=X_{1}$, which boils down to $G_{\ell}^{T} F_{\ell} X_{2} G_{m}$ $=X_{2} F_{m}$. Using $G_{\ell}^{T} F_{\ell}=J_{\ell+1}^{T}(0)$ and applying flip matrices ${ }^{1}$ from both sides we obtain the equation $J_{\ell+1}(0) X F_{m}=X G_{m}$, which only has the trivial solution according to Proposition A.1.2a and hence the interaction between both tuples is 0 .

We now show how the same result can be obtained from the tables. The setup corresponds to the third column and row "++-+" in Table 3.2. At the corresponding entry we have II:2, which means Equation II variant 2. Table 3.3 reveals that Equation II is given by $\tilde{B}_{1} \tilde{B}_{2} X \tilde{C}_{1}=X \tilde{C}_{2}$. According to Table 3.4 , variant 2 means

\footnotetext{
else.

${ }^{1}$ A flip matrix, $P_{\text {flip }}$, is a permutation matrix with ones on the anti-diagonal and zeros everywhere
} 


\begin{tabular}{|c|c|}
\hline Type & Equation \\
\hline I & $\tilde{B}_{1} \tilde{B}_{2} X=X \tilde{C}_{1} \tilde{C}_{2}$ \\
II & $\tilde{B}_{1} \tilde{B}_{2} X \tilde{C}_{1}=X \tilde{C}_{2}$ \\
III & $\tilde{B}_{1} \tilde{B}_{2} X \tilde{C}_{1} \tilde{C}_{2}=X$ \\
IV & $\tilde{B}_{1} X \tilde{C}_{1} \tilde{C}_{2}=\tilde{B}_{2} X$ \\
V & $\tilde{B}_{1} X \tilde{C}_{1}=\tilde{B}_{2} X \tilde{C}_{2}$ \\
VI & $\left\{\begin{array}{l}\tilde{B}_{1} X=\tilde{B}_{2} Y \\
X \tilde{C}_{1}=Y \tilde{C}_{2} \\
\text { VII }\end{array}\right.$ \\
VIII & $\left\{\begin{array}{l}\tilde{B}_{1} X=Y \tilde{C}_{1} \\
\tilde{B}_{2} X=Y \tilde{C}_{2} \\
\tilde{B}_{1} X=Y \tilde{C}_{1} \\
\tilde{B}_{2} Y=X \tilde{C}_{2}\end{array}\right.$ \\
\hline
\end{tabular}

Classification of matrix equation types.

the following relation between the coefficients: $\tilde{B}_{1}=B_{2}=G_{\ell}^{T}, \tilde{B}_{2}=B_{1}=F_{\ell}$, $\tilde{C}_{1}=C_{1}=F_{m}, \tilde{C}_{2}=C_{2}=G_{m}$. Moreover, the column " $\mathcal{R}_{\ell} \mathcal{R}_{m}$ " in Table 3.4 shows that the correspondingly modified pair of singular tuples is given by $\widetilde{\mathcal{B}}=\mathcal{L}_{\ell}, \widetilde{\mathcal{C}}=\mathcal{R}_{m}$. Finally, the corresponding entry in column " $\mathcal{L}_{\ell} \mathcal{R}_{m}$ " and row "II" of Table 3.5 states the interaction between the two tuples: 0 .

\begin{tabular}{|c|cccc|cccc|}
\hline Variant & $\tilde{B}_{1}$ & $\tilde{B}_{2}$ & $\tilde{C}_{1}$ & $\tilde{C}_{2}$ & $\mathcal{R}_{\ell} \mathcal{R}_{m}$ & $\mathcal{R}_{\ell} \mathcal{L}_{m}$ & $\mathcal{L}_{\ell} \mathcal{R}_{m}$ & $\mathcal{L}_{\ell} \mathcal{L}_{m}$ \\
\hline 1 & $B_{1}$ & $B_{2}$ & $C_{1}$ & $C_{2}$ & $\mathcal{R}_{\ell} \mathcal{R}_{m}$ & $\mathcal{R}_{\ell} \mathcal{L}_{m}$ & $\mathcal{L}_{\ell} \mathcal{R}_{m}$ & $\mathcal{L}_{\ell} \mathcal{L}_{m}$ \\
2 & $B_{2}$ & $B_{1}$ & $C_{1}$ & $C_{2}$ & $\mathcal{L}_{\ell} \mathcal{R}_{m}$ & $\mathcal{L}_{\ell} \mathcal{L}_{m}$ & $\mathcal{R}_{\ell} \mathcal{L}_{m}$ & $\mathcal{R}_{\ell} \mathcal{L}_{m}$ \\
3 & $B_{1}$ & $B_{2}$ & $C_{2}$ & $C_{1}$ & $\mathcal{R}_{\ell} \mathcal{L}_{m}$ & $\mathcal{R}_{\ell} \mathcal{R}_{m}$ & $\mathcal{L}_{\ell} \mathcal{L}_{m}$ & $\mathcal{L}_{\ell} \mathcal{R}_{m}$ \\
4 & $B_{2}$ & $B_{1}$ & $C_{2}$ & $C_{1}$ & $\mathcal{L}_{\ell} \mathcal{L}_{m}$ & $\mathcal{L}_{\ell} \mathcal{R}_{m}$ & $\mathcal{R}_{\ell} \mathcal{L}_{m}$ & $\mathcal{R}_{\ell} \mathcal{R}_{m}$ \\
\hline
\end{tabular}

Coefficient relation table. Depending on the matrix equation variant, this table states how the coefficients need to be modified before using Table 3.5. For example, variant 4 yields for $\mathcal{B}=\mathcal{R}_{\ell}$, $\mathcal{C}=\mathcal{R}_{m}$ the modified tuples $\widetilde{\mathcal{B}}=\mathcal{L}_{\ell}, \widetilde{\mathcal{C}}=\mathcal{L}_{m}$.

Tables 3.2-3.4 can be derived by simple but tedious algebraic manipulations. To speed up the process and reduce the risk of errors, we derived these tables using an automated procedure. In contrast, the relations in Table 3.5 are nontrivial and require further explanation.

THEOREM 3.7. The interactions stated in Table 3.5 are correct.

Proof. The proof proceeds by formulating the matrix equation corresponding to an entry in Table 3.5 and applying Proposition A.1, which states the kernel dimensions of all matrix equations needed in this process. To illustrate the process, we will 


\begin{tabular}{|c|cccc|}
\hline Equation & \multicolumn{4}{|c|}{ (Modified) Matrix Tuples } \\
\cline { 2 - 5 } Type & $\mathcal{R}_{\ell} \mathcal{R}_{m}$ & $\mathcal{R}_{\ell} \mathcal{L}_{m}$ & $\mathcal{L}_{\ell} \mathcal{R}_{m}$ & $\mathcal{L}_{\ell} \mathcal{L}_{m}$ \\
\hline I & $\min (\ell, m)$ & $\min (\ell, m+1)$ & $\min (\ell+1, m)$ & $1+\min (\ell, m)$ \\
II & 0 & $\ell$ & 0 & $\ell+1$ \\
III & 0 & 0 & 0 & 0 \\
IV & $m$ & $m+1$ & 0 & 0 \\
V & $\max (0, m-\ell)$ & $\ell+m+1$ & 0 & $\max (0, \ell-m)$ \\
VI & $\max (0, m-\ell-1)$ & $\ell+m+2$ & 0 & $\max (0, \ell-m-1)$ \\
VII & $\max (0, m-\ell+1)$ & $\ell+m$ & 0 & $\max (0, \ell-m+1)$ \\
VIII & $1+\min (\ell, m)$ & $\min (\ell, m)$ & $\min (\ell, m)$ & $1+\min (\ell, m)$ \\
\hline
\end{tabular}

Table of interactions depending on the matrix equation type (rows) and coefficients (columns). Note that the modified matrix tuples $\widetilde{\mathcal{B}}, \widetilde{\mathcal{C}}$ according to the matrix equation variant, see Table 3.4, need to be used.

prove the entries of column " $\mathcal{R}_{\ell} \mathcal{R}_{m}$ ". The following obvious relations are needed: $F_{m} G_{m}^{T}=J_{m}(0)^{T}$ and $F_{m}^{T} G_{m}=J_{m+1}(0)$.

For $\mathcal{B}=\mathcal{R}_{\ell}, \mathcal{C}=\mathcal{R}_{m}$, that is the interaction of two right singular tuples, Equation I becomes $J_{\ell}^{T}(0) X-X J_{m}^{T}(0)=0$. Here, and in the following, the transposes of Jordan blocks can be removed by applying flip matrices. According to Proposition A.1.1, the kernel of this matrix equation (and hence the interaction) is $\min (\ell, m)$. Equation II becomes $J_{\ell}^{T}(0) X F_{m}-X G_{m}=0$ and - according to Proposition A.1.2a - its kernel dimension is 0 . Equation III becomes $J_{\ell}^{T}(0) X J_{m}^{T}(0)-X=0$ and according to Proposition A.1.3 - its kernel dimension is 0. Equation IV becomes $F_{\ell} X J_{m}^{T}(0)-G_{\ell} X=0$ and - according to Proposition A.1.4a - its kernel dimension is $m$. Equation $\mathrm{V}$ becomes $F_{\ell} X F_{m}-G_{\ell} X G_{m}=0$ and - according to Proposition A.1.5a - its kernel dimension is $\max (0, m-\ell)$. Equation VI becomes $\left\{\begin{array}{l}F_{\ell} X-G_{\ell} Y=0 \\ X F_{m}-Y G_{m}=0\end{array}\right.$ and - according to Proposition A.1.6a - its kernel dimension is $\max (0, m-\ell-1)$. Equation VII becomes $\left\{\begin{array}{l}F_{\ell} X-Y F_{m}=0 \\ G_{\ell} X-Y G_{m}=0\end{array}\right.$ and - according to Proposition A.1.7a - its kernel dimension is $\max (0, m-\ell+1)$. Equation VIII becomes $\left\{\begin{array}{l}F_{\ell} X-Y F_{m}=0 \\ G_{\ell}^{T} Y-X G_{m}^{T}=0\end{array}\right.$ and - according to Proposition A.1.8a - its kernel dimension is $1+\min (\ell, m)$. $\square$

Example 3.8. As another example on how to use Tables 3.3-3.5, consider the tuples

$$
\mathcal{R}_{\ell}(2,3)=\left(I, F_{\ell}, G_{\ell}^{T}\right), \quad \mathcal{L}_{m}(1,3)=\left(F_{m}^{T}, I, G_{m}^{T}\right)
$$

with sign tuple $s=(-1,+1,+1)$. The first step is to standardize the tuples so that they conform to the conventions of the tables. We get rid of the shared third position 
by appending a fourth component with positive sign and moving $G_{m}^{T}$ to the fourth position. The standardized form of the tuples is

$$
\mathcal{B}=\mathcal{R}_{\ell}(2,3)=\left(I, F_{\ell}, G_{\ell}^{T}, I\right), \quad \mathcal{C}=\mathcal{L}_{m}(1,4)=\left(F_{m}^{T}, I, I, G_{m}^{T}\right)
$$

with sign tuple $(-1,+1,+1,+1)$. According to Table 3.2 , the corresponding matrix equation is type II variant 2. Table 3.4 reveals that variant 2 implies the use of $\mathcal{L}_{\ell} \mathcal{L}_{m}$ instead of $\mathcal{R}_{\ell} \mathcal{L}_{m}$. Finally, Table 3.5 produces the interaction $\ell+1$ for Equation II with these modified matrix tuples.

3.4. Interactions between Jordan and singular tuples. After having discussed interactions between Jordan tuples and between singular tuples in Sections 3.2 and 3.3, respectively, it remains to discuss the mixed case: interactions between Jordan and singular tuples.

Similarly to the case of interactions between singular tuples, there are several types of reduced matrix equations that may result, depending on the relative placement of the coefficients as well as the sign tuple. Similarly as in Section 3.3 we can exclude shared positions and - since the Jordan tuple contains only one nontrivial coefficient $J$ - restrict ourselves to $p=3$. For $p=3$, there are six possible relative placements, listed in the first row of Table 3.6, and there are eight possible sign combinations. The reduced matrix equations are of types I - IV in Table 3.3 with the replacements $\tilde{B}_{1} \tilde{B}_{2}=J$ and $\tilde{C}_{1} \tilde{C}_{2}=J$. Table 3.6 shows the reduced matrix equation

\begin{tabular}{|r|rr|rr|rr|}
\hline \multirow{2}{*}{ Signs } & $(J, I, I)$ & $\left(I, B_{1}, B_{2}\right)$ & $(I, J, I)$ & $\left(B_{1}, I, B_{2}\right)$ & $(I, I, J)$ & $\left(B_{1}, B_{2}, I\right)$ \\
& $\left(I, C_{1}, C_{2}\right)$ & $(J, I, I)$ & $\left(C_{1}, I, C_{2}\right)$ & $(I, J, I)$ & $\left(C_{1}, C_{2}, I\right)$ & $(I, I, J)$ \\
\hline+++ & $\mathrm{I}: 3$ & $\mathrm{I}: 2$ & $\mathrm{I}: 1$ & $\mathrm{I}: 1$ & $\mathrm{I}: 3$ & $\mathrm{I}: 2$ \\
++- & $\mathrm{II}: 1$ & $\mathrm{IV}: 1$ & $\mathrm{II}: 1$ & $\mathrm{IV}: 1$ & $\mathrm{III}: 3$ & $\mathrm{III}: 2$ \\
+-+ & $\mathrm{II}: 1$ & $\mathrm{IV}: 1$ & $\mathrm{III}: 1$ & $\mathrm{III}: 2$ & $\mathrm{II}: 1$ & $\mathrm{IV}: 1$ \\
+-- & $\mathrm{III}: 1$ & $\mathrm{III}: 2$ & $\mathrm{II}: 1$ & $\mathrm{IV}: 1$ & $\mathrm{II}: 1$ & $\mathrm{IV}: 1$ \\
-++ & $\mathrm{III}: 1$ & $\mathrm{III}: 2$ & $\mathrm{II}: 1$ & $\mathrm{IV}: 1$ & $\mathrm{II}: 1$ & $\mathrm{IV}: 1$ \\
-+- & $\mathrm{II}: 1$ & $\mathrm{IV}: 1$ & $\mathrm{III}: 1$ & $\mathrm{III}: 2$ & $\mathrm{II}: 1$ & $\mathrm{IV}: 1$ \\
--+ & $\mathrm{II}: 1$ & $\mathrm{IV}: 1$ & $\mathrm{II}: 1$ & $\mathrm{IV}: 1$ & $\mathrm{III}: 1$ & $\mathrm{III}: 1$ \\
--- & $\mathrm{I}: 1$ & $\mathrm{I}: 1$ & $\mathrm{I}: 3$ & $\mathrm{I}: 2$ & $\mathrm{I}: 1$ & $\mathrm{I}: 1$ \\
\hline
\end{tabular}

Given singular/Jordan tuples $\mathcal{B}, \mathcal{C}$ and a sign tuple $s=( \pm 1, \pm 1, \pm 1)$, this table gives the corresponding reduced matrix equation and variant.

and variant for each case of a regular tuple interacting with a singular tuple and vice versa. Note that the block $J$ is either $J_{m}(\lambda)$ with $\lambda \neq 0$ (corresponding to $\mathcal{J}_{m}(k)$ ) or $J_{m}(0)$ (corresponding to $\mathcal{N}_{m}(k)$ ). Before the interactions can be obtained, the coefficients and tuples need to be adjusted according to Table 3.7. Finally, Table 3.8 


\begin{tabular}{|c|ccc|cccc|}
\hline Variant & $J$ & $\tilde{C}_{1}$ & $\tilde{C}_{2}$ & $\mathcal{J}_{\ell} \mathcal{R}_{m}$ & $\mathcal{J}_{\ell} \mathcal{L}_{m}$ & $\mathcal{N}_{\ell} \mathcal{R}_{m}$ & $\mathcal{N}_{\ell} \mathcal{L}_{m}$ \\
\hline 1 & $J$ & $C_{1}$ & $C_{2}$ & $\mathcal{J}_{\ell} \mathcal{R}_{m}$ & $\mathcal{J}_{\ell} \mathcal{L}_{m}$ & $\mathcal{N}_{\ell} \mathcal{R}_{m}$ & $\mathcal{N}_{\ell} \mathcal{L}_{m}$ \\
3 & $J$ & $C_{2}$ & $C_{1}$ & $\mathcal{J}_{\ell} \mathcal{L}_{m}$ & $\mathcal{J}_{\ell} \mathcal{R}_{m}$ & $\mathcal{N}_{\ell} \mathcal{L}_{m}$ & $\mathcal{N}_{\ell} \mathcal{R}_{m}$ \\
\hline
\end{tabular}

\begin{tabular}{|c|ccc|cccc|}
\hline Variant & $\tilde{B}_{1}$ & $\tilde{B}_{2}$ & $J$ & $\mathcal{R}_{\ell} \mathcal{J}_{m}$ & $\mathcal{L}_{\ell} \mathcal{J}_{m}$ & $\mathcal{R}_{\ell} \mathcal{N}_{m}$ & $\mathcal{L}_{\ell} \mathcal{N}_{m}$ \\
\hline 1 & $B_{1}$ & $B_{2}$ & $J$ & $\mathcal{R}_{\ell} \mathcal{J}_{m}$ & $\mathcal{L}_{\ell} \mathcal{J}_{m}$ & $\mathcal{R}_{\ell} \mathcal{N}_{m}$ & $\mathcal{L}_{\ell} \mathcal{N}_{m}$ \\
2 & $B_{2}$ & $B_{1}$ & $J$ & $\mathcal{L}_{\ell} \mathcal{J}_{m}$ & $\mathcal{R}_{\ell} \mathcal{J}_{m}$ & $\mathcal{L}_{\ell} \mathcal{N}_{m}$ & $\mathcal{R}_{\ell} \mathcal{N}_{m}$ \\
\hline
\end{tabular}

Coefficient relation table. Depending on the matrix equation variant, this table states how the coefficients and tuples need to be modified before using Table 3.8.

gives the interaction between a Jordan and a modified singular tuple. The entries of this table follow from Proposition A.1 as in the proof of Theorem 3.7.

\begin{tabular}{|c|c|c|c|c|}
\hline \multirow{2}{*}{$\begin{array}{c}\text { Equation } \\
\text { Type }\end{array}$} & \multicolumn{4}{|c|}{ (Modified) Matrix Tuples } \\
\hline & $\mathcal{J}_{\ell} \mathcal{R}_{m}$ & $\mathcal{N}_{\ell} \mathcal{R}_{m}$ & $\mathcal{J}_{\ell} \mathcal{L}_{m}$ & $\mathcal{N}_{\ell} \mathcal{L}_{m}$ \\
\hline $\mathrm{I}$ & 0 & $\min (\ell, m)$ & 0 & $\min (\ell, m+1)$ \\
\hline II & 0 & 0 & $\ell$ & $\ell$ \\
\hline III & 0 & 0 & 0 & 0 \\
\hline
\end{tabular}

\begin{tabular}{|c|cc|cc|}
\hline Equation & \multicolumn{4}{|c|}{ (Modified) } \\
Type & $\mathcal{R}_{\ell} \mathcal{J}_{m}$ & $\mathcal{R}_{\ell} \mathcal{N}_{m}$ & $\mathcal{L}_{\ell} \mathcal{J}_{m}$ & $\mathcal{L}_{\ell} \mathcal{N}_{m}$ \\
\hline I & 0 & $\min (\ell, m)$ & 0 & $\min (\ell+1, m)$ \\
III & 0 & 0 & 0 & 0 \\
IV & $m$ & $m$ & 0 & 0 \\
\hline
\end{tabular}

Table of interactions depending on matrix equation type (rows) and modified tuples (columns).

Again we use an example to illustrate how the interaction between a singular and a Jordan tuple can be obtained from the tables above.

Example 3.9. Consider $s=(-1,-1,+1)$ and

$$
\mathcal{B}=\mathcal{R}_{\ell}(1,3)=\left(F_{\ell}, I, G_{\ell}\right), \quad \mathcal{C}=\mathcal{N}_{m}(2)=\left(I, J_{m}(0), I\right),
$$

corresponding to row "--+" and column 5 in Table 3.6. The corresponding entry states IV: 1 , referring to equation type IV variant 1 . In variant 1 , no tuples need to be modified and hence row "IV" and column " $\mathcal{R}_{\ell} \mathcal{N}_{m}$ " of Table 3.8 provides rightaway the interaction between both tuples: $m$.

As mentioned in the introduction, the generic canonical form of a rectangular 
matrix pencil $\left(p=2, s=(+1,-1), n_{2} \neq n_{1}\right)$ solely consists of singular blocks. Tables 3.6-3.8 allow to extend this statement to general $p$.

TheOREM 3.10. Let $\mathcal{A}=\left(A_{1}, \ldots, A_{p}\right)$ be a matrix tuple of the form (1.2). If the dimensions $n_{1}, \ldots, n_{p}$ do not satisfy

$$
\sum_{s_{k}=1}\left(n_{k \oplus 1}-n_{k}\right)=\sum_{s_{k}=-1}\left(n_{k}-n_{k \oplus 1}\right),
$$

then the generic canonical form of $\mathcal{A}$ solely consists of singular tuples.

Proof. Assume that $\mathcal{A}$ does not satisfy (3.7) but has a Jordan tuple $\mathcal{J}_{\ell}\left(\right.$ or $\left.\mathcal{N}_{\ell}\right)$ with $\ell \geq 1$. Since (3.7) does not hold, the canonical form of $\mathcal{A}$ must contain at least one singular tuple $\mathcal{C}$. A necessary condition for the canonical form to be generic is that there is no mutual interaction between $\mathcal{J}_{\ell}$ and any singular block $\mathcal{C}$ :

$$
d\left(\mathcal{J}_{\ell}, \mathcal{C}\right)+d\left(\mathcal{C}, \mathcal{J}_{\ell}\right)=0
$$

This can be seen from the fact that $(3.4)$ implies $c\left(\mathcal{J}_{\ell}, \mathcal{C}\right)+c\left(\mathcal{C}, \mathcal{J}_{\ell}\right)=d\left(\mathcal{J}_{\ell}, \mathcal{C}\right)+$ $d\left(\mathcal{C}, \mathcal{J}_{\ell}\right)$. Hence, if $(3.8)$ was violated, the cointeraction between $\mathcal{J}_{\ell}$ and $\mathcal{C}$ (or vice versa) would not vanish and the canonical form cannot be generic.

If $\mathcal{J}_{\ell}$ and $\mathcal{C}$ do not share a position, we can apply a cyclic permutation of the coefficients, which does not affect either interaction, such that the standardized Jordan and singular tuples take the form $(J, I, I)$ and $\left(I, C_{1}, C_{2}\right)$, respectively. This setting corresponds to columns 2 and 3 in Table 3.6. For the reduced sign tuples,++-+-+ , -+- , and --+ , the mutual interaction is $\ell$ according to rows II and IV of Table 3.8. Hence, for (3.8) to hold the reduced sign tuple must be of the form +++, +--, --or -++. In all these cases, the dimensions $m_{1}, \ldots, m_{p}$ of the corresponding singular tuple satisfy

$$
\sum_{s_{k}=1}\left(m_{k \oplus 1}-m_{k}\right)=\sum_{s_{k}=-1}\left(m_{k}-m_{k \oplus 1}\right) .
$$

To see this, first note that both $C_{1}$ and $C_{2}$ have the same sign in all of the specified sign tuples. Thus, one of the sums in (3.9) contains nothing but zeros, while the other sum contains one +1 , one -1 and otherwise only zeros.

If $\mathcal{J}_{\ell}$ and $\mathcal{C}$ share a position, we may assume without loss of generality that the tuples take the form $(J, I)$ and $\left(C_{1}, C_{2}\right)$. Applying Tables 3.6 and 3.8 to the expanded tuples shows that the mutual interaction is $\ell$ for the sign tuples,+--+ , and 0 for 0 for the sign tuples ++ and --. Note that the singular tuples satisfy (3.9) in the latter case.

In summary, all singular tuples that are admissible in the sense of (3.8) satisfy (3.9). Hence, when we remove these singular tuples from the canonical form of $\mathcal{A}$ 
then the dimensions of the resulting regular matrix tuple still violate (3.7). This is a contradiction as the coefficients of a regular tuple must be square, for which (3.7) is trivially satisfied.

Note that (3.7) is necessary but generally not sufficient in order to guarantee that $\mathcal{A}$ has a non-vanishing regular part. The following definition is motivated by the fact that (3.7) is satisfied if and only if the corresponding block cyclic embedding is a square matrix pencil, see for example [17].

Definition 3.11. A matrix tuple of the form (1.2) is called squarish if its dimension and sign tuples satisfy (3.7).

3.5. Software for counting the codimension. The results from this section have been incorporated into a Python script codimension.py that can be used to count and verify the codimension of a tuple in canonical form. The script is available at http://www.sam.math.ethz.ch/NLAgroup/codimensions.html and http://www8. cs.umu.se/ larsk/codimension.py. The canonical structure needs to be specified on input by a text file. For example, for $s=(+1,-1,+1,-1)$ and $\mathcal{A}=\mathcal{J}_{2}(1) \oplus$ $\mathcal{J}_{2}(1) \oplus \mathcal{L}_{1}(1,3) \oplus \mathcal{L}_{1}(1,4)$, this text file should take the following form:

$\mathrm{s}=(+1,-1,+1,-1)$

$\mathrm{J}(2,1)$

$\mathrm{J}(2,1)$

$\mathrm{L}(1,1,3)$

$\mathrm{L}(1,1,4)$

The script then closely follows the procedure described above to compute the interactions between all canonical tuples symbolically. The sum of these interactions yields the dimension of the kernel of the linear matrix operator (3.1) and hence the codimension of $\operatorname{orbit}(\mathcal{A})$. Additionally, the script produces a MATLAB function that constructs the matrix belonging to the Kroneckerized linear matrix operator (3.1). The kernel dimension of this matrix equals the kernel dimension of the linear operator; this automatically generated MATLAB function can therefore be used to verify the codimension of $\mathcal{A}$ numerically.

4. The special case $s=(+1, \ldots,+1)$. In principle, the results of Section 3 allow us to check whether a given canonical form is generic, simply by verifying that its codimension is zero. In practice, however, the technical complexity of the current formulation of these results gives little hope for a compact and elegant description of all generic canonical forms in the general case. The aim of this section is to point out special cases for which generic canonical forms can be obtained quite conveniently from our results. In particular, we will focus on the case $s=(+1, \ldots,+1)$. 
4.1. Contragredient equivalence: $p=2, s=(+1,+1)$. Canonical forms for a product of two matrices, i.e., $p=2$ and $s=(+1,+1)$, have received some attention in the literature, see $[12,14]$. In this special case, the equivalence relation (1.3) is sometimes called contragredient equivalence of $\left(A_{1}, A_{2}\right)$. The canonical form under contragredient equivalence is an immediate corollary of Theorem 2.1.

Corollary 4.1. Consider a matrix pair $\left(A_{1}, A_{2}\right)$ with $A_{1} \in \mathbb{C}^{n_{2} \times n_{1}}$ and $A_{2} \in$ $\mathbb{C}^{n_{1} \times n_{2}}$. Then there are invertible matrices $P_{1} \in \mathbb{C}^{n_{1} \times n_{1}}, P_{2} \in \mathbb{C}^{n_{2} \times n_{2}}$ such that $\left(P_{2}^{-1} A_{1} P_{1}, P_{1}^{-1} A_{2} P_{2}\right)$ can be written as the direct sum of pairs taking one of the following forms:
(i) $\mathcal{J}_{m}(\lambda)=\left(J_{m}(\lambda), I\right)$ with $\lambda \in \mathbb{C} \backslash\{0\}$,
(ii) $\mathcal{N}_{m}=\left(J_{m}(0), I\right)$,
(iii) $\mathcal{R}_{m}=\left(F_{m}, G_{m}^{T}\right)$,
(iv) $\mathcal{L}_{m}=\left(F_{m}^{T}, G_{m}\right)$
(nonzero Jordan pair) (zero Jordan pair) (right singular pair) (left singular pair)

This decomposition is uniquely determined up to permutation of the summands.

In the following, we count codimensions of the orbit of the matrix pair $\left(A_{1}, A_{2}\right)$ under contragredient equivalence using the general results from Section 3. For this purpose, we represent the number and sizes of the canonical pairs as follows.

- For each zero or nonzero eigenvalue $\lambda$, let $q_{1}(\lambda) \geq q_{2}(\lambda) \geq q_{3}(\lambda) \geq \cdots$ denote the decreasingly ordered sizes of its Jordan pairs.

- Let $\epsilon_{1} \geq \epsilon_{2} \geq \epsilon_{3} \geq \cdots$ denote the decreasingly ordered sizes of the right singular pairs.

- Let $\eta_{1} \geq \eta_{2} \geq \eta_{3} \geq \cdots$ denote the decreasingly ordered sizes of the left singular pairs.

Then the codimension count is

$$
c_{\text {total }}=c_{\text {Jordan }}+c_{\text {singular }}+c_{\text {Jordan }, \text { singular }},
$$

with the $c_{\text {Jordan }}, c_{\text {singular }}$ and $c_{\text {Jordan, singular }}$ depending on $q_{i}, \gamma_{i}, \epsilon_{i}, \eta_{i}$ as explained below.

Interactions between Jordan pairs. The results in Section 3.2, see in particular (3.6), imply that the total (co)interaction between Jordan pairs belonging to zero and nonzero eigenvalues is given by

$$
c_{\text {Jordan }}=\sum_{\lambda}\left(q_{1}(\lambda)+3 q_{2}(\lambda)+5 q_{3}(\lambda)+\cdots\right) .
$$

Interactions between singular pairs. Suppose that $\mathcal{B}, \mathcal{C}$ are singular pairs, i.e., $\mathcal{B} \in\left\{\mathcal{R}_{\epsilon_{i}}, \mathcal{L}_{\eta_{i}}\right\}$ and $\mathcal{C} \in\left\{\mathcal{R}_{\epsilon_{j}}, \mathcal{L}_{\eta_{j}}\right\}$. According to the procedure in Section 3.3, 
we first expand these pairs into tuples of length $p=4$ :

$$
\mathcal{B}=\left(B_{1}, I, B_{2}, I\right), \quad \mathcal{C}=\left(I, C_{1}, I, C_{2}\right), \quad s=(+1,+1,+1,+1) .
$$

Inspecting Table 3.2 we obtain matrix equation VIII variant 1 from the second column in row "++++". Since no modification of the tuples is needed for variant 1 , the interaction between $\mathcal{B}$ and $\mathcal{C}$ can be directly read off from row "VIII" of Table 3.5:

$$
\begin{aligned}
d\left(\mathcal{R}_{\epsilon_{i}}, \mathcal{R}_{\epsilon_{j}}\right) & =1+\min \left(\epsilon_{i}, \epsilon_{j}\right), & d\left(\mathcal{R}_{\epsilon_{i}}, \mathcal{L}_{\eta_{j}}\right) & =\min \left(\epsilon_{i}, \eta_{j}\right), \\
d\left(\mathcal{L}_{\eta_{i}}, \mathcal{R}_{\epsilon_{j}}\right) & =\min \left(\eta_{i}, \epsilon_{j}\right), & d\left(\mathcal{L}_{\eta_{i}}, \mathcal{L}_{\eta_{j}}\right) & =1+\min \left(\eta_{i}, \eta_{j}\right) .
\end{aligned}
$$

The cointeractions are obtained by accounting for the differences among dimensions according to (3.4):

$$
\begin{aligned}
c\left(\mathcal{R}_{\epsilon_{i}}, \mathcal{R}_{\epsilon_{j}}\right) & =\min \left(\epsilon_{i}, \epsilon_{j}\right), & c\left(\mathcal{R}_{\epsilon_{i}}, \mathcal{L}_{\eta_{j}}\right) & =1+\min \left(\epsilon_{i}, \eta_{j}\right), \\
c\left(\mathcal{L}_{\eta_{i}}, \mathcal{R}_{\epsilon_{j}}\right) & =1+\min \left(\eta_{i}, \epsilon_{j}\right), & c\left(\mathcal{L}_{\eta_{i}}, \mathcal{L}_{\eta_{j}}\right) & =\min \left(\eta_{i}, \eta_{j}\right) .
\end{aligned}
$$

Hence, the sum of all cointeractions between right singular pairs is

$$
c_{\text {right }}=\epsilon_{1}+3 \epsilon_{2}+5 \epsilon_{3}+\cdots,
$$

the sum of all cointeractions between left singular pairs is

$$
c_{\text {left }}=\eta_{1}+3 \eta_{2}+5 \eta_{3}+\cdots,
$$

and the sum of all cointeraction between left and right singular pairs as well as right and left singular pairs is

$$
c_{\text {left }, \text { right }}=2 \sum_{i, j}\left(1+\min \left\{\epsilon_{i}, \eta_{j}\right\}\right)
$$

In summary, the total cointeraction between singular pairs is $c_{\text {singular }}=c_{\text {right }}+c_{\text {left }}+$ $c_{\text {left,right }}$.

Interactions between Jordan and singular pairs. It remains to discuss the case of interactions between Jordan and singular pairs. Suppose that $\mathcal{B} \in\left\{\mathcal{J}_{q}, \mathcal{N}_{q}\right\}$ is a Jordan pair and $\mathcal{C} \in\left\{\mathcal{R}_{\epsilon_{j}}, \mathcal{L}_{\eta_{j}}\right\}$ is a singular pair. Expansion into the case $p=3$ yields the tuples

$$
\mathcal{B}=(J, I, I), \quad \mathcal{C}=\left(I, C_{1}, C_{2}\right), \quad s=(+1,+1,+1)
$$

This corresponds to equation type I variant 3 , see column 2 in row "+++" of Table 3.6.

The case of a singular pair $\mathcal{B}$ and a Jordan pair $\mathcal{C}$ is expanded into

$$
\mathcal{B}=\left(B_{1}, I, B_{2}\right), \quad \mathcal{C}=(I, J, I), \quad s=(+1,+1,+1),
$$


which corresponds to equation type I variant 1 , see column 5 in row "+++" of Table 3.6.

In the case of a nonzero eigenvalue, for a Jordan pair $\mathcal{J}_{q}$, Table 3.8 reveals zero (co)interaction. In the case of a zero eigenvalue, for a Jordan pair $\mathcal{N}_{q}$, the combined (co)interactions are given by

$$
\begin{aligned}
& c\left(\mathcal{N}_{q}, \mathcal{R}_{\epsilon_{j}}\right)+c\left(\mathcal{R}_{\epsilon_{j}}, \mathcal{N}_{q}\right)=\min \left(q, \epsilon_{j}+1\right)+\min \left(\epsilon_{j}, q\right), \\
& c\left(\mathcal{N}_{q}, \mathcal{L}_{\eta_{j}}\right)+c\left(\mathcal{L}_{\eta_{j}}, \mathcal{N}_{q}\right)=\min \left(q, \eta_{j}\right)+\min \left(\eta_{j}+1, q\right) .
\end{aligned}
$$

Hence, the total (co)interaction between Jordan pairs and singular pairs (and vice versa) is

$$
c_{\text {Jordan }, \text { singular }}=\sum_{i, j} \min \left\{2 q_{i}(0), 2 \epsilon_{j}+1\right\}+\sum_{i, j} \min \left\{2 q_{i}(0), 2 \eta_{j}+1\right\} .
$$

The generic canonical form of $\left(A_{1}, A_{2}\right)$ under contragredient equivalence. In the following, we derive the generic canonical form for a matrix pair $\left(A_{1}, A_{2}\right)$ with $s=(+1,+1)$ using the results presented above.

Consider the case $n_{2}<n_{1}$. This directly implies that the canonical form contains at least $n_{1}-n_{2}$ left singular pairs $\mathcal{L}_{\eta_{i}}$ accounting for the difference in the dimensions. An inspection of (4.2) reveals $\eta_{1}=\cdots=\eta_{n_{1}-n_{2}}=0$ in the generic case. By (4.3), the generic canonical form may not contain any right singular blocks and hence, using (4.1) and (4.4), the rest must be composed of Jordan pairs $\mathcal{J}_{1}\left(\lambda_{i}\right)$ belonging to $n_{2}$ mutually different nonzero eigenvalues $\lambda_{i}$. This yields $c_{\text {total }}=n_{2} ;$ note, however, that the codimension $n_{2}$ is compensated by the fact that the $n_{2}$ eigenvalues $\lambda_{i}$ are fixed for orbits. In summary, the generic canonical form is given by

$$
\mathcal{J}_{1}\left(\lambda_{1}\right) \oplus \cdots \oplus \mathcal{J}_{1}\left(\lambda_{n_{2}}\right) \oplus \underbrace{\mathcal{L}_{0} \oplus \cdots \oplus \mathcal{L}_{0}}_{n_{1}-n_{2} \text { times }} .
$$

Similarly in the case $n_{1}<n_{2}$, the generic canonical form is given by

$$
\underbrace{\mathcal{R}_{0} \oplus \cdots \oplus \mathcal{R}_{0}}_{n_{2}-n_{1} \text { times }} \oplus \mathcal{J}_{1}\left(\lambda_{1}\right) \oplus \cdots \oplus \mathcal{J}_{1}\left(\lambda_{n_{1}}\right) .
$$

4.2. The generic canonical form for general $p$. In the following, we derive the generic canonical form for a matrix tuple $\left(A_{1}, \ldots, A_{p}\right)$ with $s=(+1, \ldots,+1)$. With a few exceptions the arguments are quite similar to Section 4.1 ; we will therefore keep the discussion somewhat brief.

First, it is clear that the generic canonical form may only contain $1 \times 1$ Jordan tuples and singular tuples of the form $\mathcal{R}_{0}$ or $\mathcal{L}_{0}$. Moreover, the following lemma imposes some constraints on the positions of the singular blocks.

Lemma 4.2. Let $s=(+1, \ldots,+1)$. Then 


$$
\begin{array}{ll}
\text { 1. } \quad & c\left(\mathcal{R}_{0}\left(k_{1}, k_{2}\right), \mathcal{L}_{0}\left(l_{1}, l_{2}\right)\right)+c\left(\mathcal{L}_{0}\left(l_{1}, l_{2}\right), \mathcal{R}_{0}\left(k_{1}, k_{2}\right)\right)=0 \\
\Leftrightarrow \quad & k_{1}<k_{2} \leq l_{1}<l_{2} \text { or } l_{1}<l_{2} \leq k_{1}<k_{2} \text { or } k_{1}<l_{1}<l_{2}<k_{2} ; \\
\text { 2. } \quad c\left(\mathcal{R}_{0}\left(k_{1}, k_{2}\right), \mathcal{R}_{0}\left(l_{1}, l_{2}\right)\right)+c\left(\mathcal{R}_{0}\left(l_{1}, l_{2}\right), \mathcal{R}_{0}\left(k_{1}, k_{2}\right)\right)=0 \\
\Leftrightarrow \quad k_{1} \leq l_{1}<l_{2} \leq k_{2} \text { or } l_{1} \leq k_{1}<k_{2} \leq l_{2} ; \\
\text { 3. } \quad c\left(\mathcal{L}_{0}\left(k_{1}, k_{2}\right), \mathcal{L}_{0}\left(l_{1}, l_{2}\right)\right)+c\left(\mathcal{L}_{0}\left(l_{1}, l_{2}\right), \mathcal{L}_{0}\left(k_{1}, k_{2}\right)\right)=0 \\
\Leftrightarrow \quad k_{1} \leq l_{1}<l_{2} \leq k_{2} \text { or } l_{1} \leq k_{1}<k_{2} \leq l_{2} \text { or } k_{1}<k_{2}<l_{1}<l_{2} \text { or } \\
\quad l_{1}<l_{2}<k_{1}<k_{2} .
\end{array}
$$

Proof. By the reduction technique discussed in Section 3.3, we may assume without loss of generality that $p=4$. Now, to verify the statement of the lemma, only a finite number of possible block positions need to be verified. This verification has been performed using the software described in Section 3.5. The obtained results, which can be downloaded from the web page of the software, confirm the statement of the lemma.

From Lemma 4.2.1 it immediately follows that there are $n_{\min }=\min \left(n_{1}, \ldots, n_{p}\right)$ Jordan tuples $\mathcal{J}_{1}\left(\lambda_{i}\right)$ belonging to mutually different eigenvalues $\lambda_{i}$. By removing these Jordan tuples we may assume $n_{\min }=0$ for the rest of this section.

To illustrate the general procedure for obtaining the generic canonical structure, we consider the example

$$
n=(4,3,0,2,3,1)
$$

A cyclic permutation of the coefficients (which does not change the codimension) allows us to assume $n_{1}=0$ :

$$
n \leftarrow(0,2,3,1,4,3) .
$$

Having $n_{1}=0$ excludes right singular blocks, as the first dimension of $\mathcal{R}_{0}\left(k_{1}, k_{2}\right)$ is always 1 . Hence, the generic canonical form solely consists of left singular blocks $\mathcal{L}_{0}\left(k_{1}, k_{2}\right)$, whose dimensions are

$$
\left(m_{1}, \ldots, m_{p}\right)=(\underbrace{0, \ldots, 0}_{k_{1}}, \underbrace{1, \ldots, 1}_{k_{2}-k_{1}}, \underbrace{0, \ldots, 0}_{p-k_{2}}) .
$$

To determine the canonical structure we successively decompose the dimension vector $n$ into vectors of the form (4.7). Lemma 4.2 imposes some constraints on these $0 / 1$ vectors. Any two 0/1 vectors must either be nested or at least one position apart from each other. In particular, this implies that the decomposition of $n$ contains a string of consecutive 1 s for any sequence of positive integers contained in $n$. Applied to our example (4.6) this means that the decomposition will contain $(0,1,1,1,1,1)$, which corresponds to $\mathcal{L}_{0}(1,6)$. We update $n \leftarrow(0,1,2,0,3,2)$ and apply the same procedure to the longest sequence(s) of positive integers contained in $n$. Continuing this process yields the following scheme: 


\begin{tabular}{|c|cccccc|cc|c|}
\hline Step & $n_{1}$ & $n_{2}$ & $n_{3}$ & $n_{4}$ & $n_{5}$ & $n_{6}$ & $k_{1}$ & $k_{2}$ & canonical tuple \\
\hline 1 & $\mathbf{0}$ & $\mathbf{2}$ & $\mathbf{3}$ & $\mathbf{1}$ & $\mathbf{4}$ & $\mathbf{3}$ & 1 & 6 & $\rightsquigarrow \mathcal{L}_{0}(1,6)$ \\
2 & $\mathbf{0}$ & $\mathbf{1}$ & $\mathbf{2}$ & 0 & 3 & 2 & 1 & 3 & $\rightsquigarrow \mathcal{L}_{0}(1,3)$ \\
3 & 0 & 0 & 1 & $\mathbf{0}$ & $\mathbf{3}$ & $\mathbf{2}$ & 4 & 6 & $\rightsquigarrow \mathcal{L}_{0}(4,6)$ \\
4 & 0 & 0 & 1 & $\mathbf{0}$ & $\mathbf{2}$ & $\mathbf{1}$ & 4 & 6 & $\rightsquigarrow \mathcal{L}_{0}(4,6)$ \\
5 & 0 & $\mathbf{0}$ & $\mathbf{1}$ & 0 & 1 & 0 & 2 & 3 & $\rightsquigarrow \mathcal{L}_{0}(2,3)$ \\
6 & 0 & 0 & 0 & $\mathbf{0}$ & $\mathbf{1}$ & 0 & 4 & 5 & $\rightsquigarrow \mathcal{L}_{0}(4,5)$ \\
\hline & 0 & 0 & 0 & 0 & 0 & 0 & & & \\
\hline
\end{tabular}

The general procedure for $n=\left(n_{1}, \ldots, n_{p}\right)$ with $n_{1}=0$ is as follows.

While $n \neq 0$

1. Find a longest sequence $n_{k_{1}+1}, n_{k_{1}+2}, \ldots, n_{k_{2}}$ of positive integers.

2. Add the left singular tuples $\mathcal{L}_{0}\left(k_{1}, k_{2}\right)$ to the generic canonical form.

3. Update $n_{k_{1}+1} \leftarrow n_{k_{1}+1}-1, n_{k_{1}+2} \leftarrow n_{k_{1}+2}-1, \ldots, n_{k_{2}} \leftarrow n_{k_{2}}-1$.

\section{End While}

It is easy to check that any two tuples $\mathcal{L}_{0}\left(k_{1}, k_{2}\right), \mathcal{L}_{0}\left(k_{1}^{\prime}, k_{2}^{\prime}\right)$ generated by this procedure satisfy the conditions of Lemma 4.2.3. Therefore the obtained canonical structure is generic. Once the procedure has been completed, we need to apply the inverse of the cyclic permutation, which was used to guarantee $n_{1}=0$, to the canonical tuples. This process is straightforward and shall only be illustrated with our example. The generic canonical structures of the tuples belonging to the permuted and original dimensions (4.6) and (4.5), respectively, take the following form:

$$
\begin{aligned}
& (0,2,3,1,4,3) \\
& \left(F_{0}^{T}, I_{1}, I_{1}, I_{1}, I_{1}, G_{0}\right) \quad\left(I_{1}, G_{0}, F_{0}^{T}, I_{1}, I_{1}, I_{1}\right) \quad \mathcal{R}_{0}(2,3) \\
& \oplus\left(F_{0}^{T}, I_{1}, G_{0}, I_{0}, I_{0}, I_{0}\right) \quad \oplus\left(I_{0}, I_{0}, F_{0}^{T}, I_{1}, G_{0}, I_{0}\right) \quad \oplus \mathcal{L}_{0}(3,5) \\
& \oplus\left(I_{0}, I_{0}, I_{0}, F_{0}^{T}, I_{1}, G_{0}\right) \leadsto \oplus\left(I_{1}, G_{0}, I_{0}, I_{0}, I_{0}, F_{0}^{T}\right) \quad \hat{=} \oplus \mathcal{R}_{0}(2,6) \\
& \oplus\left(I_{0}, I_{0}, I_{0}, F_{0}^{T}, I_{1}, G_{0}\right) \quad \oplus\left(I_{1}, G_{0}, I_{0}, I_{0}, I_{0}, F_{0}^{T}\right) \quad \oplus \mathcal{R}_{0}(2,6) \\
& \oplus\left(I_{0}, F_{0}^{T}, G_{0}, I_{0}, I_{0}, I_{0}\right) \quad \oplus\left(I_{0}, I_{0}, I_{0}, F_{0}^{T}, G_{0}, I_{0}\right) \quad \oplus \mathcal{L}_{0}(4,5) \\
& \oplus\left(I_{0}, I_{0}, I_{0}, F_{0}^{T}, G_{0}, I_{0}\right) \quad \oplus\left(G_{0}, I_{0}, I_{0}, I_{0}, I_{0}, F_{0}^{T}\right) \quad \oplus \mathcal{R}_{0}(1,6)
\end{aligned}
$$

As already mentioned above, a cyclic permutation will not change the codimension. Indeed, any two singular tuples from $\mathcal{R}_{0}(2,3) \oplus \mathcal{L}_{0}(3,5) \oplus \mathcal{R}_{0}(2,6) \oplus \mathcal{R}_{0}(2,6) \oplus \mathcal{L}_{0}(4,5) \oplus$ $\mathcal{R}_{0}(1,6)$ satisfy the conditions of Lemma 4.2 and the canonical form is therefore still of codimension 0 .

5. Orthogonal reduction to square factors. Numerical algorithms, such as the periodic QZ algorithm [1, 11], for solving the periodic eigenvalue problem asso- 
ciated with a matrix tuple are not able to handle nonsquare coefficients directly. To circumvent this restriction, we develop a numerically stable procedure for extracting the square, generically regular part from a tuple. After having performed this reduction, existing algorithms can be applied to the extracted square part.

For $s=(+1, \ldots,+1)$, Section 4.2 shows that the generically regular square part has order $n_{\min }=\min \left\{n_{1}, \ldots, n_{p}\right\}$. In general, this is not the case. In particular, if the matrix tuple is not squarish in the sense of (3.7), then Theorem 3.10 shows that the generically regular square part has order 0 independent of $n_{\min }$. To avoid this degenerate situation, we will assume that the matrix tuple is squarish:

$$
\sum_{s_{k}=+1}\left(n_{k \oplus 1}-n_{k}\right)=\sum_{s_{k}=-1}\left(n_{k}-n_{k \oplus 1}\right) .
$$

Unfortunately, as we will see in Example 5.3 below, even this assumption does not imply that the generically regular square part has order $n_{\min }$.

The reduction procedure to be described in the following has two stages, aimed at reducing factors with $s_{k}=1$ and $s_{k}=-1$, respectively, to square form.

Stage 1. To illustrate the idea of Stage 1 of the proposed extraction procedure, we will first discuss a rather detailed example.

ExAmple 5.1. Consider a matrix tuple for $p=6$ with dimension tuple $n=$ $(3,5,4,5,5,2)$ and sign tuple $s=(+1,-1,+1,-1,+1,-1)$. Then the generalized product $A_{6}^{-1} A_{5} A_{4}^{-1} A_{3} A_{2}^{-1} A_{1}$ takes the shape pictured below.

0)

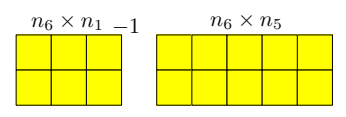

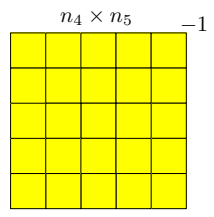
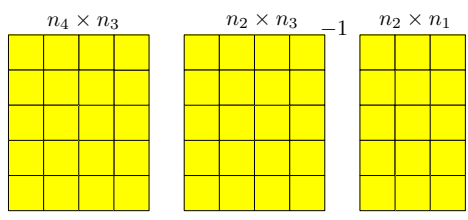

The first step of the reduction procedure consists of compressing the $n_{2}=5$ rows of $A_{1}$ using a $\mathrm{QR}$ factorization, which results in an upper trapezoidal matrix with a generically nonsingular $r_{2} \times r_{1}$ block in the upper left corner $\left(r_{1}=r_{2}=3\right)$.
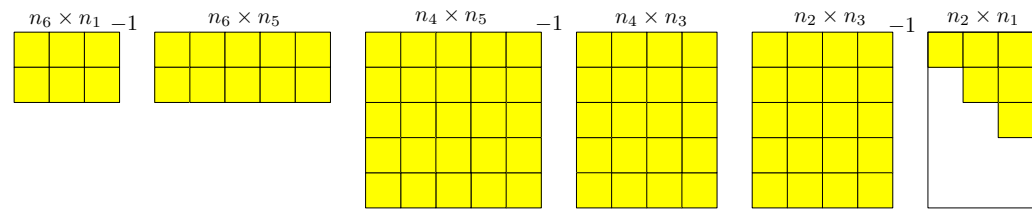

To obtain an equivalence transformation of the entire tuple, we have to pre-multiply $A_{2}$ by the orthogonal matrix from the QR factorization. Immediately after this update, the two $\left(n_{2}-r_{2}\right)$ bottom rows of $A_{2}$ are compressed by an RQ factorization 
and $A_{3}$ is updated correspondingly.

1)
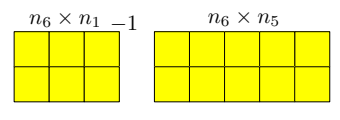
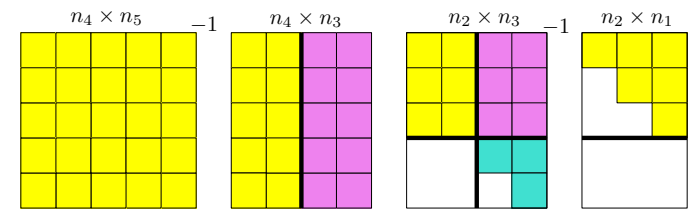

The thick lines illustrate the $2 \times 2$ block upper triangular structure of each block of $\mathcal{A}$. The tuple corresponding to the upper left block has the dimensions $r=(3,3,2,5,5,2)$ while the lower right block has the dimension $n-r=(0,2,2,0,0,0)$. Note that some of the subblocks are degenerate in the sense of having one dimension equal to zero. Only the upper left tuple may contain any regular part and is therefore processed further. We continue compressing the two leading columns of $A_{3}$ to a $2 \times 2$ matrix using a $\mathrm{QR}$ factorization. After the corresponding update of $A_{4}$, we compress the three bottom rows of $A_{3}$ using an RQ factorization.

2)
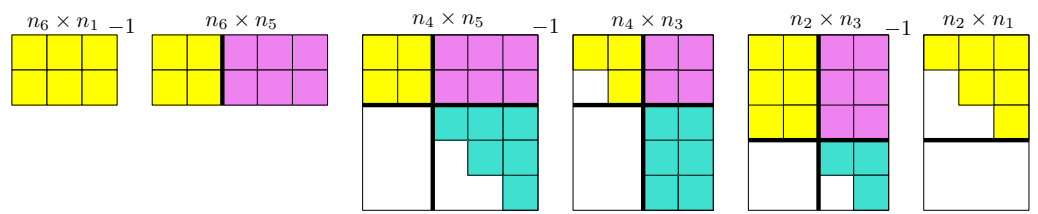

Note that the remaining unreduced block tuple has dimensions $(3,3,2,2,2,2)$ and all its coefficients with $s_{k}=1$ are square.

It is instructive to describe the procedure in Example 5.1 purely in terms of integer operations on the dimension vector $n=\left(n_{1}, \ldots, n_{p}\right)$. For this purpose, we define

$$
\Delta_{k}=\left\{\begin{array}{ll}
n_{k \oplus 1}-n_{k}, & \text { if } s_{k}=1, \\
n_{k}-n_{k \oplus 1}, & \text { if } s_{k}=-1,
\end{array} \quad \Delta^{+}=\sum_{s_{k}=+1} \Delta_{k}, \quad \Delta^{-}=\sum_{s_{k}=-1} \Delta_{k}\right.
$$

LEMMA 5.2. $\Delta^{+}=-\Delta^{-}$.

Proof. We have

$$
\Delta=\sum_{k=1}^{p}\left(n_{k \oplus 1}-n_{k}\right)=\sum_{k=1}^{p} n_{k \oplus 1}-\sum_{k=1}^{p} n_{k}=0
$$

which, combined with $\Delta^{+}+\Delta^{-}=\Delta$, implies the result.

For a squarish tuple, (5.1) is equivalent to $\Delta^{+}=\Delta^{-}$, which combined with Lemma 5.2 implies

$$
\Delta^{+}=\Delta^{-}=0 .
$$


0)

\begin{tabular}{l|cccccc}
$k$ & 1 & 2 & 3 & 4 & 5 & 6 \\
$s_{k}$ & + & - & + & - & + & - \\
\hline$r_{k}$ & 3 & 5 & 4 & 5 & 5 & 2 \\
$r_{k \oplus 1}$ & 5 & 4 & 5 & 5 & 2 & 3 \\
\hline$\Delta_{k}^{(0)}$ & 2 & 1 & 1 & 0 & -3 & -1 \\
\hline$n_{k}-r_{k}$ & 0 & 0 & 0 & 0 & 0 & 0
\end{tabular}

1)

\begin{tabular}{l|cccccc}
$k$ & 1 & 2 & 3 & 4 & 5 & 6 \\
$s_{k}$ & + & - & + & - & + & - \\
\hline$r_{k}$ & 3 & $\mathbf{3}$ & $\mathbf{2}$ & 5 & 5 & 2 \\
$r_{k \oplus 1}$ & $\mathbf{3}$ & $\mathbf{2}$ & 5 & 5 & 2 & 3 \\
\hline$\Delta_{k}^{(1)}$ & $\mathbf{0}$ & 1 & $\mathbf{3}$ & 0 & -3 & -1 \\
\hline$n_{k}-r_{k}$ & 0 & $\mathbf{2}$ & $\mathbf{2}$ & 0 & 0 & 0
\end{tabular}

2)

\begin{tabular}{l|cccccc}
$k$ & 1 & 2 & 3 & 4 & 5 & 6 \\
$s_{k}$ & + & - & + & - & + & - \\
\hline$r_{k}$ & 3 & 3 & 2 & $\mathbf{3}$ & $\mathbf{2}$ & 2 \\
$r_{k \oplus 1}$ & 3 & 2 & $\mathbf{2}$ & $\mathbf{3}$ & 2 & 3 \\
\hline$\Delta_{k}^{(2)}$ & 0 & 1 & $\mathbf{0}$ & 0 & $\mathbf{0}$ & -1 \\
\hline$n_{k}-r_{k}$ & 0 & 2 & 2 & $\mathbf{3}$ & $\mathbf{3}$ & 0
\end{tabular}

FIG. 5.1. Table of dimension vectors and indices belonging to Example 5.1: 0) initial table, 1) after first reduction step, 2) after second reduction step.

EXAMPLE 5.1 CTD. To interpret the reductions performed in Example 5.1 as operations on the dimensions, let $r=\left(r_{1}, \ldots, r_{p}\right)$ denote the dimensions of the unreduced tuple which remains to be processed.

0) Initially, $r_{k}=n_{k}$ and $\Delta_{k}^{(0)}=\Delta_{k}$ with $\Delta_{k}$ defined in (5.2). This initial configuration is shown in Figure 5.1 (0).

1) In the first step of the reduction procedure, $A_{1}$ is reduced to a square matrix: $r_{2} \leftarrow r_{2}-\Delta_{1}^{(0)}=r_{1}$ and $\Delta_{1}^{(1)}=0$. A $\Delta_{1}^{(0)} \times \Delta_{1}^{(0)}$ diagonal block is split off from $A_{2}: r_{3} \leftarrow r_{3}-\Delta_{1}^{(0)}$. This does not affect $\Delta_{2}^{(0)}$ but $\Delta_{3}^{(0)}$ increases to

$$
\Delta_{3}^{(1)}=r_{4}-r_{3}=n_{4}-\left(n_{3}-\Delta_{1}\right)=\Delta_{1}+\Delta_{3} .
$$

The configuration after the first reduction step is shown in Figure 5.1 (1), with the modified quantities in bold face.

2) The next step consists of reducing $A_{3}$ to a square matrix $\left(r_{4} \leftarrow r_{4}-\Delta_{3}^{(1)}=r_{3}\right.$ and $\left.\Delta_{3}^{(2)}=0\right)$ and splitting off a correspondingly sized diagonal block from $A_{4}\left(r_{5} \leftarrow r_{5}-\Delta_{3}^{(1)}\right)$. Note that $\Delta_{5}^{(1)}$ increases to

$$
\Delta_{5}^{(2)}=r_{6}-r_{5}=n_{6}-\left(n_{5}-\Delta_{3}^{(1)}\right)=\Delta_{1}+\Delta_{3}+\Delta_{5},
$$

using (5.4). The configuration after the second reduction step is shown in Figure 5.1 (2).

At this point the reduction of Stage 1 is complete as $\Delta_{k}^{(2)}=0$ for all $k$ with $s_{k}=+1$. 
Let us generalize the procedure described in Example 5.1. Suppose $t-1$ reduction steps have been successfully completed and our aim is to reduce the next factor $A_{k_{t}}$ with $s_{k_{t}}=+1$ to a square matrix. Letting $k_{t+1}$ denote the smallest index with $k_{t+1}>k_{t}$ and $s_{k_{t+1}}=+1$, the corresponding dimension table takes the following form:

\begin{tabular}{l|lcccccc}
$k$ & $\ldots$ & $k_{t}$ & $k_{t}+1$ & $\ldots$ & $k_{t+1}-1$ & $k_{t+1}$ & $\ldots$ \\
$s_{k}$ & $\ldots$ & + & - & $\ldots$ & - & + & $\cdots$ \\
\hline$r_{k}$ & $\cdots$ & $r_{k_{t}}$ & $r_{k_{t}+1}$ & $\cdots$ & $r_{k_{t+1}-1}$ & $r_{k_{t+1}}$ & $\cdots$ \\
$r_{k \oplus 1}$ & $\cdots$ & $r_{k_{t}+1}$ & $r_{k_{t}+2}$ & $\ldots$ & $r_{k_{t+1}}$ & $r_{k_{t+1} \oplus 1}$ & $\cdots$ \\
\hline$\Delta_{k}^{(t-1)}$ & $\cdots$ & $\Delta_{k_{t}}^{(t-1)}$ & $\Delta_{k_{t}+1}^{(t-1)}$ & $\cdots$ & $\Delta_{k_{t+1}-1}^{(t-1)}$ & $\Delta_{k_{t+1}}^{(t-1)}$ & $\cdots$
\end{tabular}

Provided that $\Delta_{k_{t}}^{(t-1)} \geq 0$ the reduction step $t$ consists of a QR factorization of $A_{k_{t}}$ and RQ factorizations of $A_{k_{t}+1}, \ldots, A_{k_{t+1}-1}$, affecting the dimensions as follows:

$$
\begin{aligned}
r_{k_{t}+1} & \leftarrow r_{k_{t}+1}-\Delta_{k_{t}}^{(t-1)}=r_{k_{t}}, \\
r_{k_{t}+2} & \leftarrow r_{k_{t}+2}-\Delta_{k_{t}}^{(t-1)}, \\
& \vdots
\end{aligned} \quad \Delta_{k}^{(t)}= \begin{cases}0, & \text { if } k=k_{t}, \\
\Delta_{k_{t}+1}^{(t-1)}+\Delta_{k_{t}}^{(t-1)}, & \text { if } k=k_{t+1}, \\
\Delta_{k_{t}+1}^{(t-1)}, & \text { otherwise. }\end{cases}
$$

Two things can go wrong at this point.

Breakdown 1 It is not admissible to perform the subtraction with $\Delta_{k_{t}}^{(t-1)}$ on the left side of (5.5) if $r_{\min }<\Delta_{k_{t}}^{(t-1)}$ for $r_{\min }=\min \left\{r_{k_{t}+2}, \ldots, r_{k_{t+1}}\right\}$. However, in this case there is no regular block left. More specifically, if $r_{\min } \leq \Delta_{k_{t}}^{(t-1)}$ we can use RQ factorizations to split off $r_{\min } \times r_{\min }$ diagonal blocks from $A_{k_{t}+1}, \ldots, A_{k_{t+1}-1}$ such that the remaining tuple has at least one dimension zero and is therefore singular.

Breakdown 2 When $\Delta_{k_{t+1}}^{(t)}<0$ after the update (5.5), the subsequent reduction step cannot be performed. This situation can be avoided as follows. Define

$$
\sigma_{k}:=\sum\left\{\Delta_{\ell}: \ell \leq k, s_{\ell}=+1\right\}
$$

Then (5.5) implies $\sigma_{k_{t}}=\Delta_{k_{t}}^{(t)}$ by induction. Let $k^{\prime}$ be such that $\sigma_{k^{\prime}}=\min \sigma_{k}$. If $\sigma_{k^{\prime}}<0$ we perform the following cyclic permutation

$$
\pi:\left(1, \ldots, k^{\prime}-1, k^{\prime}, k^{\prime}+1, \ldots, p\right) \mapsto\left(k^{\prime}+1, \ldots, p, 1, \ldots, k^{\prime}-1, k^{\prime}\right)
$$

to the factors:

$$
\tilde{A}_{j}=A_{\pi(j)}, \quad \tilde{s}_{j}=s_{\pi(j)} .
$$

For the permuted product, $\sigma_{p}$ becomes minimal among all $\sigma_{k}$. Note that (5.3) implies $\sigma_{p}=0$ and therefore all $\sigma_{k}$ become nonnegative:

$$
\Delta_{k_{t}}^{(t)}=\sigma_{k_{t}} \geq 0
$$


In the following, we will assume that the cyclic permutation described above has already been performed and (5.6) is satisfied. Let $k_{1}, \ldots, k_{\mathrm{pl}}$ denote all $k$ with $s_{k}=$ +1 . Provided that Breakdown 1 does not occur, $\mathrm{pl}-1$ reduction steps (5.5) yield $\Delta_{k_{1}}^{(\mathrm{pl}-1)}=\cdots=\Delta_{k_{\mathrm{pl}-1}}^{(\mathrm{pl}-1)}=0$. In addition, (5.3) implies $\sigma_{p}=\Delta_{k_{\mathrm{pl}}}^{(\mathrm{pl}-1)}=0$. In other words, all factors $A_{k}$ with $s_{k}=+1$ have been reduced to square form.

Algorithm 1 realizes the procedure described above.

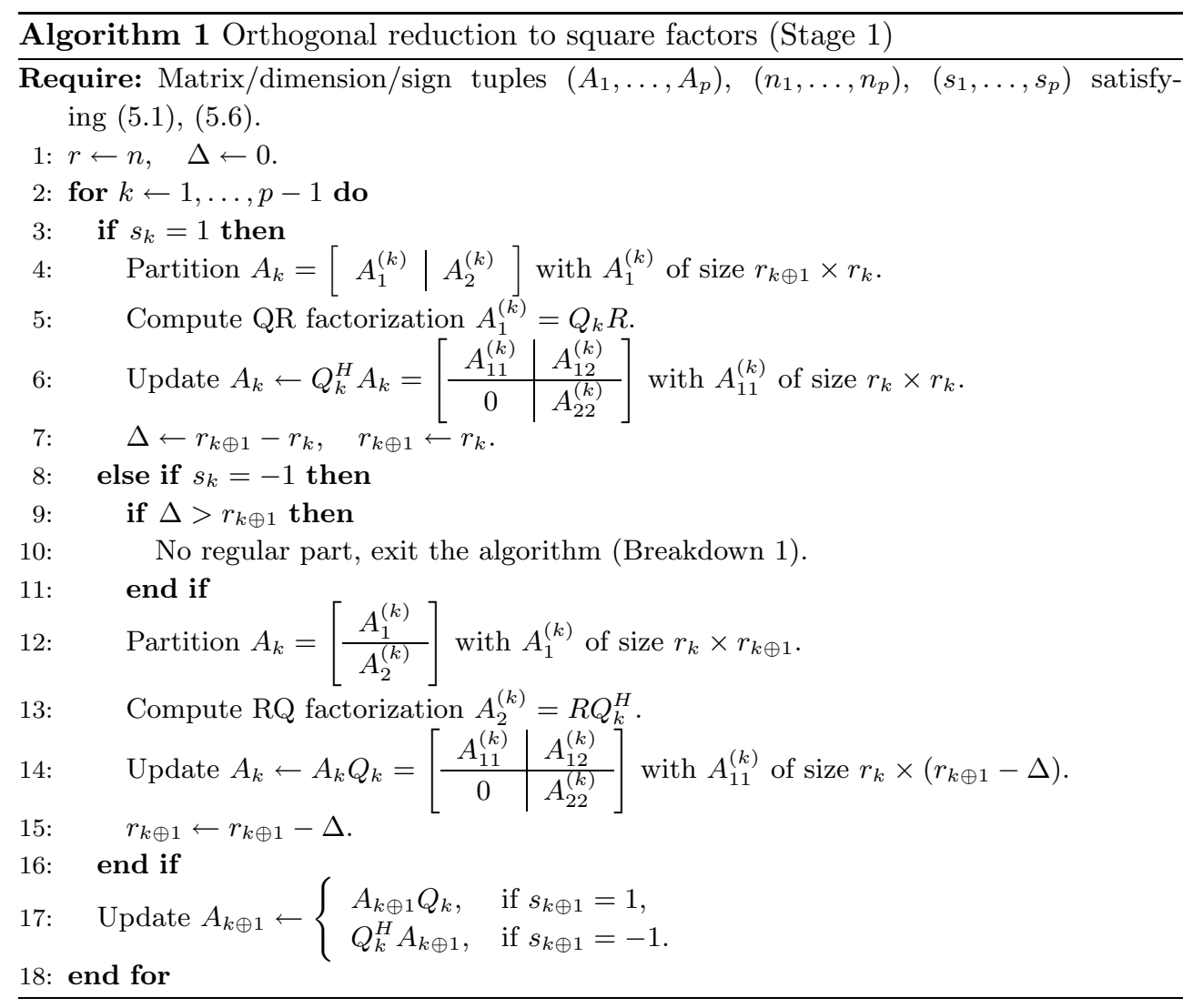

Stage 2. Let $\left(A_{1}, \ldots, A_{p}\right)$ be the tuple obtained from a successful completion of Stage 1, i.e.,

$$
A_{k}=\left[\begin{array}{c|c}
A_{11}^{(k)} & A_{12}^{(k)} \\
\hline 0 & A_{22}^{(k)}
\end{array}\right], \quad A_{11}^{(k)} \in \mathbb{C}^{r_{k \oplus 1} \times r_{k}}
$$

with $r_{k \oplus 1}=r_{k}$ for $s_{k}=+1$. To reduce the factors with $s_{k}=-1$ to square form a process very similar to Algorithm 1 can be used. Instead of giving a formal description we feel that it is sufficient to illustrate the algorithm for two examples. 
EXAMPLE 5.1 CTD. Stage 1 has resulted in $r=(3,3,2,2,2,2)$ :
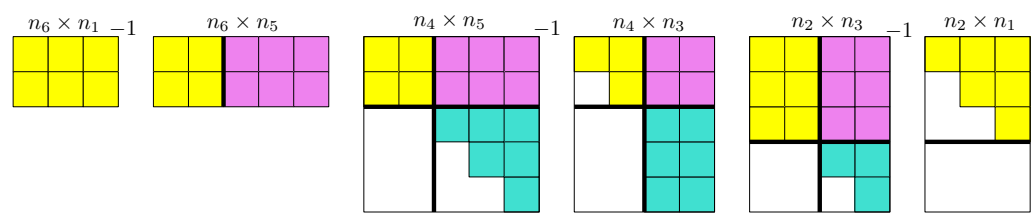

Stage 2 proceeds by applying an RQ factorization to $A_{6}$, annihilating its first column. After updating $A_{1}$, we (re)compute its $\mathrm{QR}$ decomposition and obtain the following picture.
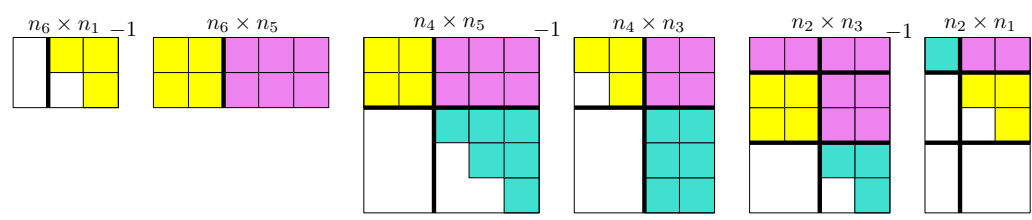

Hence, a singular tuple of dimensions $(1,1,0,0,0,0)$ splits off at the top left corner and the remaining middle tuple is square and of order 2 .

ExAmPle 5.3. Finally, Figure 5.2 illustrates Stages 1 and 2 for a matrix tuple with $n=(3,5,4,3,5,4)$ and $s=(+1,-1,-1,-1,+1,+1)$. While $n_{\min }=3$, the obtained reduced square part has only order 1 .

6. Conclusions and open questions. In the first part of the paper, we have provided formulas and software for computing the codimension of a generalized matrix product in canonical form. These formulas have been used to describe and prove the generic canonical form in the special case of a standard matrix product. In the most general case, however, the complexity of the formulas does not admit a compact and elegant description of the generic canonical form. It is presently not clear to us whether there is an algebraic framework admitting such a description.

In the second part of the paper, we have - motivated by the results from the first part - derived an orthogonal reduction algorithm for extracting square submatrices from a generalized matrix product with rectangular factors. A product with square coefficients is generically regular and admits the application of existing numerical algorithms and software. The described algorithm is part of a larger effort to develop a software package for computing eigenvalues and deflating subspaces of generalized matrix products $[9,10]$.

\section{Appendix A. Kernel dimensions of singular matrix equations.}

Proposition A.1. Let the matrices $J_{m}(\lambda), F_{m}, G_{m}$ be defined as in (1.1). Then the following statements hold. 

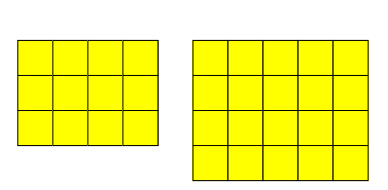

\section{Stage 1}
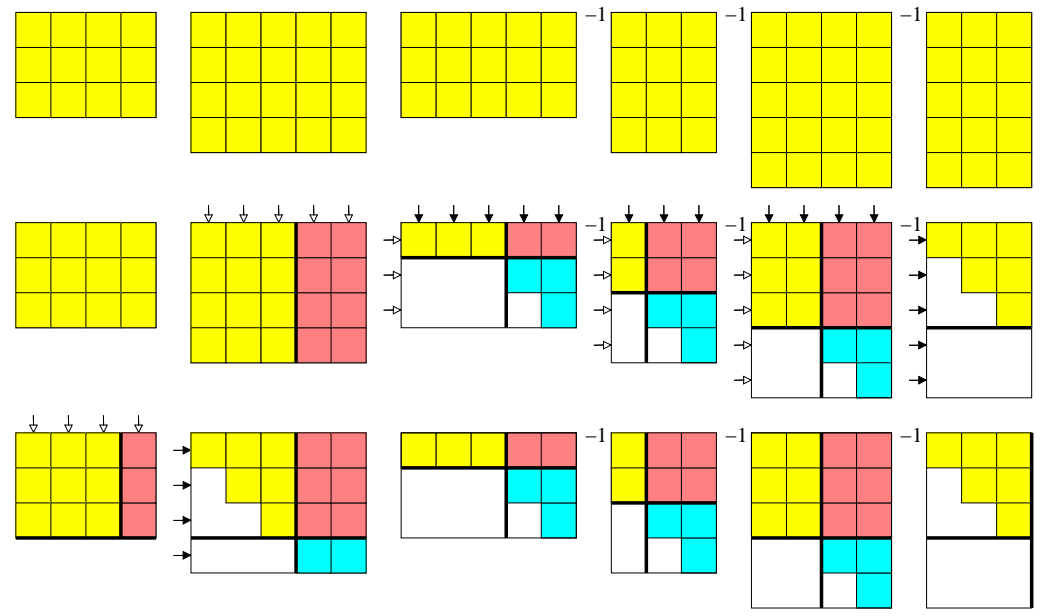

Stage 2
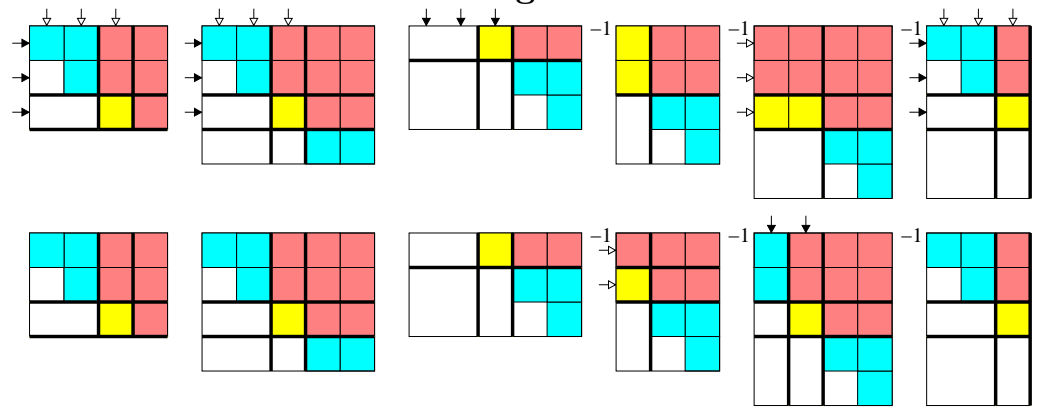

FIG. 5.2. Stages 1 and 2 applied to the generalized matrix product $A_{6} A_{5} A_{4}^{-1} A_{3}^{-1} A_{2}^{-1} A_{1}$ in Example 5.3. Solid arrows indicate rows/columns affected by a reduction and hollow arrows indicate rows/columns affected by an update.

1. $\operatorname{dim}\left\{X: J_{\ell}(\lambda) X-X J_{m}(\lambda)=0\right\}=\min \{\ell, m\}$;

2. (a) $\operatorname{dim}\left\{X: J_{\ell}(\lambda) X F_{m}-X G_{m}=0\right\}=0$,

(b) $\operatorname{dim}\left\{X: J_{\ell}(\lambda) X F_{m}^{T}-X G_{m}^{T}=0\right\}=\ell$;

3. $\operatorname{dim}\left\{X: J_{\ell}(0) X J_{m}(\lambda)-X=0\right\}=0$ for every $\lambda \in \mathbb{C}$;

4. (a) $\operatorname{dim}\left\{X: F_{\ell} X J_{m}(\lambda)-G_{\ell} X=0\right\}=m$,

(b) $\operatorname{dim}\left\{X: F_{\ell}^{T} X J_{m}(\lambda)-G_{\ell}^{T} X=0\right\}=0$;

5. (a) $\operatorname{dim}\left\{X: F_{\ell} X G_{m}-G_{\ell} X F_{m}=0\right\}=\max \{0, m-\ell\}$,

(b) $\operatorname{dim}\left\{X: F_{\ell} X G_{m}^{T}-G_{\ell} X F_{m}^{T}=0\right\}=\ell+m+1$,

(c) $\operatorname{dim}\left\{X: F_{\ell}^{T} X G_{m}-G_{\ell}^{T} X F_{m}=0\right\}=0$,

(d) $\operatorname{dim}\left\{X: F_{\ell}^{T} X G_{m}^{T}-G_{\ell}^{T} X F_{m}^{T}=0\right\}=\max \{0, \ell-m\}$;

6. (a) $\operatorname{dim}\left\{(X, Y): F_{\ell} X-G_{\ell} Y=0, X F_{m}-Y G_{m}=0\right\}=\max \{0, m-\ell-1\}$,

(b) $\operatorname{dim}\left\{(X, Y): F_{\ell} X-G_{\ell} Y=0, X F_{m}^{T}-Y G_{m}^{T}=0\right\}=\ell+m+2$, 
(c) $\operatorname{dim}\left\{(X, Y): F_{\ell}^{T} X-G_{\ell}^{T} Y=0, X F_{m}-Y G_{m}=0\right\}=0$,

(d) $\operatorname{dim}\left\{(X, Y): F_{\ell}^{T} X-G_{\ell}^{T} Y=0, X F_{m}^{T}-Y G_{m}^{T}=0\right\}=\max \{0, \ell-m-1\}$;

7. (a) $\operatorname{dim}\left\{(X, Y): F_{\ell} X-Y F_{m}=0, G_{\ell} X-Y G_{m}=0\right\}=\max \{0, m-\ell+1\}$,

(b) $\operatorname{dim}\left\{(X, Y): F_{\ell} X-Y F_{m}^{T}=0, G_{\ell} X-Y G_{m}^{T}=0\right\}=\ell+m$,

(c) $\operatorname{dim}\left\{(X, Y): F_{\ell}^{T} X-Y F_{m}=0, G_{\ell}^{T} X-Y G_{m}=0\right\}=0$,

(d) $\operatorname{dim}\left\{(X, Y): F_{\ell}^{T} X-Y F_{m}^{T}=0, G_{\ell}^{T} X-Y G_{m}^{T}=0\right\}=\max \{0, \ell-m+1\}$;

8. (a) $\operatorname{dim}\left\{(X, Y): F_{\ell} X-Y F_{m}=0, G_{\ell}^{T} Y-X G_{m}^{T}=0\right\}=1+\min \{\ell, m\}$,

(b) $\operatorname{dim}\left\{(X, Y): F_{\ell} X-Y F_{m}^{T}=0, G_{\ell}^{T} Y-X G_{m}=0\right\}=\min \{\ell, m\}$,

(c) $\operatorname{dim}\left\{(X, Y): F_{\ell}^{T} X-Y F_{m}=0, G_{\ell} Y-X G_{m}^{T}=0\right\}=\min \{\ell, m\}$,

(d) $\operatorname{dim}\left\{(X, Y): F_{\ell}^{T} X-Y F_{m}^{T}=0, G_{\ell} Y-X G_{m}=0\right\}=1+\min \{\ell, m\}$.

Proof.

1. This result is well known and can be found, e.g., in the book by Gantmacher [7].

2. (a) From

$$
J_{\ell}(\lambda) X F_{m}-X G_{m}=J_{\ell}(\lambda)[X, 0]-[0, X]=0
$$

it follows that the last column of $X$ is zero, from which it follows that the previous last column of $X$ is zero, and so on. Hence, only $X=0$ is an admissible solution.

(b) From

$$
J_{\ell}(\lambda) X F_{m}^{T}-X G_{m}^{T}=J_{\ell}(\lambda)\left[X e_{1}, \ldots, X e_{m}\right]-\left[X e_{2}, \ldots, X e_{m+1}\right]=0
$$

it follows that the first column of $X$ can be freely chosen. After this choice, all the other columns of $X$ are determined: $X e_{2}=J_{m}(\lambda) X e_{1}$, $X e_{3}=J_{m}(\lambda) X e_{2}$ and so on. Hence, the dimension of the kernel is $\ell$, the number of rows in $X$.

3. Since the eigenvalues of $J_{\ell}(0)$ are not the reciprocals of the eigenvalues of $J_{m}(\lambda)$, the Stein equation $J_{\ell}(0) X J_{m}(\lambda)-X$ has the unique solution $X=0$, see, e.g., [13].

4. By transposition, equation $F_{\ell} X J_{m}(\lambda)-G_{\ell} X=0$ is turned into $J_{m}^{T}(\lambda) X^{T} F_{\ell}^{T}$ $-X^{T} G_{\ell}^{T}=0$. By the same arguments as for $2 \mathrm{~b}$, the dimension of the kernel is $m$. Analogously, it follows as in 2a that $F_{\ell}^{T} X J_{m}(\lambda)-G_{\ell}^{T} X=0$ has the unique solution $X=0$.

5. (a) The equation $F_{\ell} X G_{m}-G_{\ell} X F_{m}=0$ in terms of the entries $x_{i j}, i=$ $1, \ldots, \ell+1, j=1, \ldots, m$, is equivalent to

$$
\begin{aligned}
x_{i+1,1}=0, \quad i=1, \ldots, \ell \\
x_{i, j}-x_{i+1, j+1}=0, \quad i=1, \ldots, \ell, j=1, \ldots, m-1 \\
x_{i, m}=0, \quad i=1, \ldots, \ell
\end{aligned}
$$


The equations (A.2) say that $X$ is a Toeplitz matrix, i.e., its entries are constant along the diagonals. Then (A.1) and (A.3) imply that the outermost $\ell$ diagonals in the top right and in the bottom left parts are zero. However, $X$ has only $\ell+m$ diagonals in total and therefore $m \leq \ell$ implies $X=0$. If $m>\ell$ there remain $m-\ell$ diagonals in the middle to be freely chosen and hence the dimension of all $X$ satisfying the equation is $m-\ell$.

(b) The equation $F_{\ell} X G_{m}^{T}-G_{\ell} X F_{m}^{T}=0$ in terms of the entries $x_{i j}, i=$ $1, \ldots, \ell+1, j=1, \ldots, m+1$, gives

$$
x_{i, j+1}-x_{i+1, j}=0, \quad i=1, \ldots, \ell, j=1, \ldots, m,
$$

which is equivalent to saying that $X$ is a Hankel matrix, i.e., $X$ is constant along the antidiagonals. The dimension of all $(\ell+1) \times(m+1)$ Hankel matrices is $\ell+m+1$.

(c) From

$$
0=F_{\ell}^{T} X G_{m}-G_{\ell}^{T} X F_{m}=\left[\begin{array}{cc}
0 & X \\
0_{11} & 0
\end{array}\right]-\left[\begin{array}{cc}
0 & 0_{11} \\
X & 0
\end{array}\right]
$$

it follows that the first row/column and the last row/column of $X$ are zero, which implies that also the second row/column and the previous last row/column are zero, and so on, eventually yielding $X=0$.

(d) This result follows from 5 a after transposing the equation.

6. (a) Partition $X=\left[x_{1}, \tilde{X}\right]$ and $Y=\left[\tilde{Y}, y_{m}\right]$. Then $X F_{m}-Y G_{m}=0$ decomposes into $x_{1}=y_{m}=0$ and $\tilde{X}=\tilde{Y}$. In other words, $X=$ $\tilde{X} G_{m-1}$ and $Y=\tilde{X} F_{m-1}$. Inserting this into $F_{\ell} X-G_{\ell} Y=0$ gives $F_{\ell} \tilde{X} G_{m-1}-G_{\ell} \tilde{X} F_{m-1}=0$ and hence the result follows from $5 \mathrm{a}$.

(b) Partition $X=\left[\tilde{X}, x_{m+1}\right]$ and $Y=\left[y_{1}, \tilde{Y}\right]$. Then $X F_{m}^{T}-Y G_{m}^{T}=\tilde{X}-$ $\tilde{Y}=0$. In other words, $X=\hat{X} G_{m+1}^{T}$ and $Y=\hat{X} F_{m+1}^{T}$ with $\hat{X}=$ $\left[y_{1}, \tilde{X}, x_{m+1}\right]$. Inserting this into $F_{\ell} X-G_{\ell} Y=0$ gives $F_{\ell} \hat{X} G_{m+1}^{T}-$ $G_{\ell} \hat{X} F_{m+1}^{T}=0$ and hence the result follows from $5 \mathrm{~b}$.

(c) This result follows, similarly as 6a, from 5 a.

(d) This result follows from 6 a after transposing the equation.

7. These results can be found in [3, Sec. 5].

8. (a) Partition $X=\left[\begin{array}{cc}\tilde{X} & c \\ r & x_{\ell+1, m+1}\end{array}\right]$, then the equation $F_{\ell} X-Y F_{m}$ decomposes into $Y=\tilde{X}$ and $c=0$. Hence, the second equation $G_{\ell}^{T} Y-X G_{m}^{T}$ turns into $G_{\ell}^{T} \tilde{X}-X G_{m}^{T}$. Adding a zero column to $G_{\ell}^{T}$ gives $\left[G_{\ell}, 0\right]=$ $J_{\ell+1}^{T}(0)$ and we obtain the equivalent equation $J_{\ell+1}^{T}(0)\left[\begin{array}{c}\tilde{X} \\ r\end{array}\right]-X G_{m}^{T}=0$. Adding the trivially satisfied equation $J_{\ell+1}^{T}(0)\left[\begin{array}{c}c \\ x_{\ell+1, m+1}\end{array}\right]-X \cdot 0=0$ to this equation yields $J_{\ell+1}^{T}(0) X-X J_{m+1}^{T}(0)=0$. Applying 1 to the 
transpose of the latter equation shows that the dimension of all such $X$ is $\min \{\ell+1, m+1\}=1+\min \{\ell, m\}$.

(b) The equation $G_{\ell}^{T} Y-X G_{m}$ is equivalent to $Y=[0, \tilde{X}], X=\left[\begin{array}{c}0 \\ \tilde{X}\end{array}\right]$ for some $\ell \times m$ matrix $\tilde{X}$. The first equation $F_{\ell} X-Y F_{m}^{T}=0$ thus reduces to $J_{\ell}(0)^{T} \tilde{X}-\tilde{X} J_{m}(0)=0$. This is equivalent to $J_{\ell}(0)\left(P_{\text {flip }} \tilde{X}\right)-$ $\left(P_{\text {flip }} \tilde{X}\right) J_{m}(0)=0$, where $P_{\text {flip }}$ denotes the flip matrix. Hence, the result follows from 1.

(c) This result follows from $8 \mathrm{~b}$ after transposing both equations.

(d) This result follows from 8a after transposing both equations.

\section{REFERENCES}

[1] A. Bojanczyk, G.H. Golub, and P. Van Dooren. The periodic Schur decomposition; algorithm and applications. Proceedings of SPIE Conference, 1770:31-42, 1992.

[2] F. De Terán and F.M. Dopico. A note on generic Kronecker orbits of matrix pencils with fixed rank. SIAM J. Matrix Anal. Appl., 30(2):491-496, 2008.

[3] J.W. Demmel and A. Edelman. The dimension of matrices (matrix pencils) with given Jordan (Kronecker) canonical forms. Linear Algebra Appl., 230:61-87, 1995.

[4] J.W. Demmel and B. Kågström. The generalized Schur decomposition of an arbitrary pencil $A-\lambda B$ : robust software with error bounds and applications. I. Theory and algorithms. ACM Trans. Math. Software, 19(2):160-174, 1993.

[5] J.W. Demmel and B. Kågström. The generalized Schur decomposition of an arbitrary pencil $A-\lambda B$ : robust software with error bounds and applications. II. Software and applications. ACM Trans. Math. Software, 19(2):175-201, 1993.

[6] P. Donovan and M.R. Freislich. The Representation Theory of Finite Graphs and Associated Algebras. Carleton Mathematical Lecture Notes, No. 5. Carleton University, Ottawa, Ontario, 1973.

[7] F.R. Gantmacher. The Theory of Matrices. Chelsea, New York, 1960.

[8] G. H. Golub and C.F. Van Loan. Matrix Computations, third edition. Johns Hopkins University Press, Baltimore, 1996.

[9] R. Granat, B. Kågström, and D. Kressner. Computing periodic deflating subspaces associated with a specified set of eigenvalues. BIT Numerical Mathematics, 47(4):763-791, 2007.

[10] R. Granat, B. Kågström, and D. Kressner. Matlab tools for solving periodic eigenvalue problems. In G. Leonov and A. Fradkov, editors, Third IFAC Workshop on Periodic Control Systems, 2007.

[11] J.J. Hench and A.J. Laub. Numerical solution of the discrete-time periodic Riccati equation. IEEE Trans. Automat. Control, 39(6):1197-1210, 1994.

[12] O. Holtz. Applications of the duality method to generalizations of the Jordan canonical form. Linear Algebra Appl., 310(1-3):11-17, 2000.

[13] R.A. Horn and C.R. Johnson. Topics in Matrix Analysis. Cambridge University Press, Cambridge, 1991.

[14] R.A. Horn and D.I. Merino. Contragredient equivalence: a canonical form and some applications. Linear Algebra Appl., 214:43-92, 1995.

[15] S. Iwata and R. Shimizu. Combinatorial analysis of singular matrix pencils. SIAM J. Matrix Anal. Appl., 29(1):245-259, 2007.

[16] B. Kågström. Singular matrix pencils. In Z. Bai, J.W. Demmel, J.J. Dongarra, A. Ruhe, and H. van der Vorst, editors, Templates for the Solution of Algebraic Eigenvalue Problems. SIAM, Philadelphia, PA, 2000. 
[17] D. Kressner. The periodic QR algorithm is a disguised QR algorithm. Linear Algebra Appl., 417(2-3):423-433, 2006.

[18] L.A. Nazarova. Representations of quivers of infinite type. Izv. Akad. Nauk SSSR Ser. Mat., 37:752-791, 1973.

[19] C. Schröder. URV decomposition based structured methods for palindromic and even eigenvalue problems. Preprint 375, Matheon, 2007.

[20] V.V. Sergeichuk. Canonical matrices for linear matrix problems. Linear Algebra Appl., 317(13):53-102, 2000.

[21] V.V. Sergeichuk. Computation of canonical matrices for chains and cycles of linear mappings. Linear Algebra Appl., 376:235-263, 2004.

[22] P. Van Dooren. The Generalized Eigenstructure Problem. PhD Thesis, Electronic Sciences Lab. - USCEE Report 503, University of Southern California, 1979.

[23] C.F. Van Loan. Generalized Singular Values with Algorithms and Applications. PhD Thesis, The University of Michigan, 1973.

[24] A. Varga. Balancing related methods for minimal realization of periodic systems. Systems Control Lett., 36(5):339-349, 1999.

[25] A. Varga. Computation of Kronecker-like forms of periodic matrix pairs. Proceedings of Sixteenth International Symposium on Mathematical Theory of Networks and Systems (MTNS 2004), Leuven, Belgium, 2004.

[26] A. Varga. On solving discrete-time periodic Riccati equations. Proceedings of 16th IFAC World Congress, Prague, 2005.

[27] A. Varga and P. Van Dooren. Computational methods for periodic systems - an overview. Proceedings of IFAC Workshop on Periodic Control Systems, Como, Italy, 171-176, 2001.

[28] W.C. Waterhouse. The codimension of singular matrix pairs. Linear Algebra Appl., 57:227-245, 1984. 